\title{
DAMAGE AND STRAIN LOCALIZATION AROUND A PRESSURIZED SHALLOW-LEVEL MAGMA RESERVOIR
}

Jean-Luc Got, ${ }^{1}$ David Amitrano, ${ }^{2}$ Ioannis Stefanou, ${ }^{3}$ Elodie Brothelande, ${ }^{4}$

Aline Peltier ${ }^{5}$

Corresponding author: J.-L. Got, ISTerre, Université Savoie Mont Blanc, 73376, Le Bourgetdu-Lac FRANCE. (jlgot@univ-savoie.fr)

${ }^{1}$ Université Savoie Mont Blanc, Université

Grenoble Alpes, CNRS, IRD, IFSTTAR,

ISTerre, F-73376 Le Bourget-du-Lac, France

${ }^{2}$ Université Grenoble Alpes, Université

Savoie Mont Blanc, CNRS, IRD, IFSTTAR,

ISTerre, F-38406, Saint-Martin d'Héres,

France

${ }^{3}$ Ecole des Ponts ParisTech, Laboratoire

NAVIER, F-75009, Paris, France

${ }^{4}$ Department of Terrestrial Magnetism,

Carnegie Institution for Science,

Washington DC, USA 
Abstract. Structures developing above long-term growing shallow-level magma reservoirs, such as resurgent domes, may contain information on the reservoir itself. To understand the formation of such tectonic features, we have investigated the deformation process around a shallow pressurized magma reservoir embedded in a damaging elastic volcanic edifice. Our model allows evidencing the effect of the progressive damage in producing the fault pattern associated to tectonic surface deformation. Damage is first isotropic around the cavity and constitutes a damaged zone. Then the free-surface effect appears and an anisotropic shear strain develops from the boundary of the damaged zone; it localizes on reverse faults that propagate upward to the surface. When the surface deformation is sufficient, normal faulting appears. Finally, the complete structure shows an undeformed wedge above the damaged zone. This structure is similar to those found by analogue modelling and from field geologic observations. From this model, we found a relation to estimate the reservoir radius and depth from the graben and dome widths. From limit analysis, we deduced an analytical expression of the magma reservoir pressure which provides a better understanding of the magma pressure build-up during doming. The dip of reverse faults limiting the dome can be

\footnotetext{
${ }^{5}$ Observatoire Volcanologique du Piton de la Fournaise, IPGP, Sorbonne Paris Cité, UMR 7154, CNRS, Paris, France
} 
inferred from the minimal pressure required to rupture the crust around the reservoir. Finally, the magma reservoir overpressure, the dip of the faults, the reservoir depth and the damaged zone radius is inferred from three parameters: the ratio $\rho_{R}$ computed from the dome and graben widths, the cohesion and the friction angle. 


\section{Introduction}

The location of most volcanoes is related to large tectonic structures controlled by geodynamics. These structures are created by regional horizontal tectonic stresses: for instance, rifts or cordilleras are created by tensile or compressive stresses, respectively.

At the volcano scale, some remarkable structures like resurgent domes result from the pressurization of shallow-level magma reservoirs, which provides a vertical component to the stress field. Retrieving the geometrical and some physical characteristics of the pressure source from geological and geophysical surface observations, especially taking into account the faulting structure, is a challenge that requires the development of realistic models involving strain localization.

Resurgent domes are commonly observed in the central part of large active calderas. They are found, for example, at Yellowstone, Long Valley, Lake Toba, Valles Caldera (e.g. Smith and Bailey [1968]), Siwi Caldera, Campi Flegrei [Sacchi et al., 2014]. Caused by the long-term uplift of the caldera floor, they are attributed to renewed magma intrusion after the caldera collapse and subsequent pressurization of a shallow-level magma reservoir. Most resurgent domes are elongated in shape along the same elongation direction of their hosting caldera, they are therefore mostly 2D structures.

The apex of the domes is generally occupied by one longitudinal graben (in rare cases, such as in Toba or Long Valley, domes can bear several parallel grabens). Normal faults forming this graben are well identified at the surface and have been studied at depth by geothermal drillings in the case of Redondo dome in Valles Caldera (Figure 1) or seismic imaging at Campi Flegrei [Sacchi et al., 2014]. The nature of dome borders is 
22

less well understood, as they are not associated to outcropping faults identified at the surface. However, they represent a sharp transition between the dome flanks and the flat caldera moat, strongly suggesting that the dome lateral extension could be limited by reverse faults. This hypothesis is corroborated by the evidence of fluid circulation along borders. Multiple geyser basins (including the renown Old Faithful) concentrate along the border of Mallard Lake resurgent dome in Yellowstone caldera [Christiansen, 2001]. Electrical surveys in the Siwi caldera also evidenced probable hydrothermal circulation along the Yenkahe dome border [Brothelande et al., 2016a]. Dome borders also represent a preferential pathway for magmatic fluids, as late volcanic products seem to commonly originate from there. Examples include late cinder cones along the Yenkahe (Figure 1), late rhyolite around Long Valley resurgent dome (moat rhyolite; Bailey and Dalrymple [1976]), Redondo dome in Valles Caldera (ring domes, Figure 1; Smith and Bailey [1968]), and La Pacana resurgent dome [Gardeweg and Ramirez, 1987; Lindsay et al., 2001].

To understand the structural evolution leading to the building of such resurgent domes, analogue experiments have been conducted [Sanford, 1959; Marti et al., 1994; Merle and Vendeville, 1995; Acocella et al., 2000, 2001; Galland et al., 2009; Brothelande and Merle, 2015]. The models revealed the presence of reverse faults limiting the dome, and the ones using elongated sources could reproduce the development of longitudinal grabens, as observed in natural domes (Figure S1).

The question of the expansion of a cylindrical or spherical cavity has been extensively studied since the beginning of the twentieth century, first in an infinite or semi-infinite elastic medium (see, e.g., Jeffery [1920]). This has led to an improved understanding of the structures around magma reservoirs and the development of the first mechanical models 
45

46

47

of pressurized magmatic structures (Anderson [1936]; Sanford [1959]; Robson and Barr [1964]; Phillips [1974]; see, e.g., Tibaldi [2015] for a review). However, modelling faulting around a pressurized cavity requires taking into account the plastic deformation to localize strain. In this aim, a considerable amount of work has been produced in mechanics and geomechanics to model the stresses and displacement around over- or underpressurized cavities in linear elastic, perfectly plastic materials, with various plastic flow rules (see, e.g., Nadai [1931]; Hill et al. [1947]; Hill [1950]; Nadai [1950]; Chadwick [1959]; Salencon [1966]; Vesic [1972]; D'Escatha and Mandel [1974]; Carter et al. [1986]; Sulem et al. [1987];

Vardoulakis et al. [1988]; Bigoni and Laudiero [1989]; Yu [2000]; see Bigoni and Laudiero [1989] for a historical introduction of this question).

Numerical modelling of volcano-tectonic structures around a pressurized magma reservoir in an elasto-plastic semi-infinite medium was first made by Grosfils [2007] and Gerbault et al. [2012], and by Brothelande et al. [2016b] in the case of resurgent domes. This later model leads to phenomenological relations between source parameters and surface observables. However, elasto-plastic modelling is closely linked to mechanical instability and subject to numerical instabilities that may prevent from modelling the complete faulting geometry, especially when the magma reservoir is overpressurized and close to the free surface of a semi-infinite medium. In this work we first derive a stable representation of the faulting geometry by using continuum damage mechanics. Continuum damage mechanics is a widely used approach in mechanics and geomechanics for modelling strain localization before rupture through progressive change of mechanical properties (see, e.g., Kachanov [1958]; Lemaitre [1994]; Krajcinovic [1996]; Lyakhovsky et al. [1997]; Main [2000]; de Borst [2002]; Turcotte and Glasscoe [2004]; Amitrano and Helmstetter [2006]; Heap et al. [2010]). 
It has been used to model the progressive landslide failure [Eberhardt et al., 2004; Lacroix and Amitrano, 2013], and more recently to model the dynamics of basaltic volcanoes preeruptive deformation and seismicity [Carrier et al., 2015; Got et al., 2017]. In the present work our aim was to get a complete representation of the faulting geometry around a pressurized reservoir by continuum damage mechanics, in order to use it to establish geometrical and physical relations between source parameters (reservoir overpressure, depth and radius, fault dip), surface observables (graben and dome widths) and rock mass parameters (friction angle and cohesion), by using limit analysis. Limit analysis is also a widely used method to provide analytical or numerical relations in stability problems (see, e.g., Davis and Selvadurai [2002] as a simple and good reference textbook on the subject) and may be applied to volcanological mechanical questions.

\section{Model}

We investigated the damage process and its consequence for the dome growth by defining an initially axisymmetric, homogeneous, and isotropic two-dimensional model which represents a shallow-level pressurized magma reservoir in a damageable elastic half-space (Figure 2). Discretizing this model in finite elements, we compute the plane strain deformation using roller boundary conditions along the model vertical borders, and fixed boundary conditions at the bottom. Surface is free. Model was scaled to physical dimensions (Figure 2), and set to a $10 \mathrm{~km} \times 10 \mathrm{~km}$ square, with an initial linear elastic medium containing an initial $0.5 \mathrm{~km}$ radius circular central cavity. A uniform pressure is applied along the boundary of the cavity all along the numerical experiment. Gravity is applied to each node, using a rock density $\rho=2700 \mathrm{~kg} / \mathrm{m}^{3}$, a value comprised between andesite 
89

$\left(\rho=2600 \mathrm{~kg} / \mathrm{m}^{3}\right)$ and basalt $\left(\rho=2900 \mathrm{~kg} / \mathrm{m}^{3}\right)$ rock densities in the $0-25 \mathrm{~km}$ depth interval

(see, e.g., Christensen and Mooney [1999], Table 4).

In this modelling we use the finite element progressive damage approach of Amitrano et al. [1999]; Amitrano [2003]; Amitrano and Helmstetter [2006], previously used in Lacroix and Amitrano [2013] and more recently in Riva et al. [2018]. It allows the reproduction of the progressive failure of rock by using an elastic damage model. It follows Kachanov [1958]'s elastic damage approach in which the Young's modulus $E_{i}$ of each element $i$ decreases when a failure criterion is met and a fracture occurs. Damage onset has been shown to follow a Mohr-Coulomb criterion [Heap et al., 2009], which has been chosen as the instantaneous damage threshold by Amitrano and Helmstetter [2006], Lacroix and Amitrano [2013] and in the present model. This Mohr-Coulomb criterion is truncated when the normal stress is equal to the tensile strength. Progressive damage induces the progressive decrease of the Young's modulus with the number of damage events; for one damage event:

$$
E_{i}(n+1)=(1-\delta) E_{i}(n)
$$

or, for $n$ damage events:

$$
E_{i}(n)=(1-\delta)^{n} E_{0}
$$

where $\delta$ is the incremental damage occurring during one damage event, and $E_{0}$ is the initial Young's modulus. Heterogeneity in the model is introduced by using a random perturbation of the Young's modulus spatial distribution.

Stress redistribution occurs around a damaged element and may induce an avalanche of damage events. The number of damaged elements is the avalanche size, which may be thought as a measure of its magnitude. 
Damage also occurs as a subcritical process, on elements where the applied shear stress is lower than the instantaneous, maximal, strength. These elements are located outside the zone of maximum damage. In that case, damage is delayed and described by a law determined from static fatigue experiments, which expresses the time to failure $t_{f}$ as a function of the major principal stress $\sigma_{1}$ and of the instantaneous compressive or tensile strength $\sigma_{0}$ [Wiederhorn and Bolz, 1970; Das and Scholz, 1981]:

$$
t_{f}=t_{0} \exp \left(-b \frac{\sigma_{1}}{\sigma_{0}}\right)
$$

The time $t_{0}$ and the constant $b$ depend on rock properties and ambient conditions $[$ Scholz, 1972]. They are scaling parameters for the dynamics of the rupture process. Each element $i$ is characterized by its failure time $t_{i}$ without interaction between elements (initially $t_{i}=t_{f}$ ), and the proportion of consumed lifetime [Amitrano and Helmstetter, 2006], which allows taking into account the stress history for estimating the remaining time to failure. Taking into account this subcritical crack growth process and the random part in the spatial distribution of Young's modulus perturbations avoids an instantaneous localization in a very narrow fault plane. It allows the dissipation of the elastic potential energy outside this plane and widens the damaged zone, creating heterogeneity in this zone. It contributes to regularize the strain localization process, spreading damage in time and space, and controlling its time dynamics. Therefore, in the absence of data (deformation, seismicity), the information on physical processes operating during damage is contained in the regularization process; it is the physical a priori knowledge of this process.

As a consequence, in this model, the pressure applied in the reservoir is constant, but larger than the long-term strength of the rock mass, so that earthquakes occur around the 
reservoir and weaken the volcanic edifice. This weakening occurs through the progressive decrease of the Young's modulus; it may induce strain localization when stress conditions are sufficient and remain unchanged. In this work, we will not perform a parametric study that would allow us to find phenomenological relations; we will instead search for a faulting geometry and use limit analysis to find physical analytical relations between source parameters and observables.

\section{Results and discussion}

\subsection{Strain localization and spatial distribution of damage}

At the beginning of the pressurization process (Figure 3 (a)), a damaged shell appears around the pressurized reservoir, and isotropic strain localization develops along more or less radial features around the reservoir. These features correspond to the logarithmic spirals that are well observed when an infinite isotropic elastic-plastic medium is plastified under the pressure applied in a hole or cylindrical cavity (see, e.g., Hill [1950]; D'Escatha and Mandel [1974]; Davis and Selvadurai [2002]; Gerbault et al. [2012]) when no spatial regularization is applied. During this initial phase, the free surface is not perturbed and the external boundary of the damaged zone is quasi-circular, as it is in the case of an infinite medium. Hill [1950] gives an expression for the radius of the damaged zone around a pressurized reservoir in an infinite medium:

$$
\frac{r_{D Z}}{r}=\left(\frac{2 E}{3 Y}\right)^{\frac{1}{3}}
$$

where $r_{D Z}$ is the radius of the damaged zone, $r$ is the radius of the cavity, $E$ is the Young's modulus and $Y$ is the yield stress of the rock mass. Taking a value of $5 \mathrm{GPa}$ for $E$ and 
$10 \mathrm{MPa}$ for $Y$, in the order of magnitude of those for an average quality rock mass (see, e.g., Hoek and Brown [1997]), gives $r_{D Z} \approx 6 r$, which is close to our result.

However our model is not infinite. Large superficial deformation develops after isotropic damage occurs around the reservoir (Figure 3); it appears to be a free-surface effect. It produces an anisotropic strain component that dominates the isotropic component outside the damaged zone. Free surface and damage induce the punching failure of the rock mass above the pressurized cavity. Damaged elements delimit zones where anisotropic shear strain progressively localizes, and forms a coherent and complex system of faults with reverse and normal faulting. Reverse faults $\Phi_{r}$ progressively delimit an uplifted compartment similar to resurgent domes (see, e.g., Brothelande et al. [2016b]). These faults initiate first at depth, at the external boundary of the damaged zone, at some distance of its top, and progress towards the surface. Their average dip is about $60^{\circ}$. Normal faults $\Phi_{n}$ develop at the surface when the dome progressively builds; they delimit a central graben, which is a frequently observed feature in such structures. A characteristic undeformed wedge, delimited by faults $\Phi_{w}$ is present above the top of the damaged zone. Though the pressure source is not located at the surface, this undeformed wedge shares strong similarities with the well-known Prandtl's wedge for shallow strip footings in geomechanics (see, e.g., Davis and Selvadurai [2002]). These results show that the final damage spatial distribution reproduces quite accurately the results of published geological field observations (see, e.g., Merle et al. [2013]) and analogue modelling (Sanford [1959], Davison et al. [1993], Marti et al. [1994], Merle and Vendeville [1995], Acocella et al. [2001], Merle et al. [2013] and Brothelande and Merle [2015]): resurgent domes are structures with reverse faulting on their external boundaries and internal normal faulting (see, e.g., Acocella et al. 
so that

$$
B=\frac{W_{D}-W_{G}}{2}
$$


192

and

$$
W_{D}=B+H \cot \beta
$$

where $\beta$ is the dip of the reverse fault $\phi_{r}$; from the latter equation we deduce:

$$
H=\frac{W_{D}+W_{G}}{2} \tan \beta
$$

By the Jeffery's points we can draw an infinity of circles, one only being tangent to the straight lines issued from the point I (Figure 4). Considering that the reservoir center C is located at the intersection of the normal to these straight lines, we find the depth of this center:

$$
h_{c}=\left(1+\left(\frac{B}{H}\right)^{2}\right) H
$$

and the radius of the damaged zone:

$$
r_{D Z}=B\left(1+\left(\frac{B}{H}\right)^{2}\right)^{\frac{1}{2}}
$$

If the reservoir section is not circular, $h_{c}$ is the depth of the center of curvature of the damaged zone top, with the curvature taken at Jeffery's points. We notice that the ratio:

$$
\lambda=\frac{H}{B}=\frac{W_{D}+W_{G}}{W_{D}-W_{G}} \tan \beta
$$

may be known only from field data, so that $h_{c}$ may be written:

$$
h_{c}=\left(1+\left(\frac{W_{D}-W_{G}}{W_{D}+W_{G}} \cot \beta\right)^{2}\right)\left(\frac{W_{D}+W_{G}}{2}\right) \tan \beta
$$

One can also express the reservoir radius $r_{c}$ as a function of the damaged zone radius:

$$
r_{D Z}=(1+\Delta) r_{c}
$$

where $\Delta$ is the relative damage zone thickness (close to 5 in our case), so that $r_{c}$ may be written:

$$
\left.r_{c}=\frac{W_{D}-W_{G}}{2(\text { Jahury }}\left(1+2 \frac{W_{D}-W_{G}}{28,} \operatorname{dW\Phi ,+3W18pm}\right)^{2}\right)^{\frac{1}{2}}
$$




\subsection{Magma reservoir pressure derived from Limit Analysis}

In such a model, when the geometry of damaged zones and the displacement field are known, limit analysis theorems (see, e.g., Davis and Selvadurai [2002], Souloumiac et al. [2009]) give us the possibility to compute an upper bound for the pressure in the magma reservoir. The aim of this approach is to provide an analytical expression for the magma reservoir pressure, to understand which are the control parameters of the pressurization process and what is the relation with the deformation process.

The upper bound theorem of Limit Analysis states that failure will occur if, for any kinematically admissible displacement field, the rate of work of the external forces is equal to or exceeds the rate of energy dissipation. It allows us to determine the maximum, ultimate value (upper bound) of the applied external loads that a system can bear without collapsing, which in our case corresponds to the loss of equilibrium. For collapse modes of translational type, the upper bound is equivalent to the solution found by the limit equilibrium method (see, e.g., Drescher and Detournay [1993]): the upper bound theorem expresses the energy balance of the limit load, which is equivalent to the limit equilibrium of the system on the base of the principle of virtual powers.

Results of the modelling allow us to identify the blocks that are sliding and to deduce their relative velocities (Figure 4). This type of collapse mode is of translational type and therefore the limit equilibrium method can be used as well. However, the upper bound theorem is easier to apply given the geometric complexity of the system. Applying the upper bound theorem requires computing the rate of the work of external forces (weights and pressure force) and the rate of the dissipation. In that aim we need to compute the relative velocities of the sliding blocks; they are found by constructing the hodograph 
(see Appendix 1, Figure S2), taking into account the angle of dilatancy $\psi$. We can then express the directions of the velocities, and find the modules by projecting the Chasles's relation on each coordinates axis (see Appendix 1 for detailed calculations). Given that there is no horizontal displacement of the vertical boundaries, the horizontal forces do not work. The rate of work may therefore be found by computing the weights of the various sliding blocks (see Appendix 1):

$$
W_{e x t}=-\left(2 \rho g H B v_{01}+w_{2} v_{02}\right)+2 P B v_{01}
$$

Applying the upper bound theorem, that is, writing that the rate of external work of the external forces is equal to the rate of internal energy dissipation $D$ leads to find the pressure:

$$
P=\rho g H+\frac{w_{2} v_{02}}{2 B v_{01}}+\frac{D}{2 B v_{01}}
$$

that may be written explicitely as (see Appendix 1 for the detailed computation of $w_{2} v_{02}$ and $D)$ :

$$
\begin{aligned}
P=\rho g H+\frac{1}{4} \rho g B \tan \beta\left(\frac{\lambda}{\tan \beta}-1\right)^{2}(1 & \left.-\frac{\tan (\beta-\psi)}{\tan (\beta+\psi)}\right) \\
& +2 C \cos \phi \frac{\lambda \cos ^{2} \beta \cos \psi+\sin ^{2} \beta \sin \psi}{\sin 2 \beta \sin (\beta+\psi) \cos (\beta-\psi)}
\end{aligned}
$$

where $\lambda=H / B$ and $C$ is the cohesion. In the general case where a deviatoric stress exists at the depth $H$, the initial lithostatic pressure may be expressed as a fraction of the vertical stress:

$$
P_{0}=k \rho g H
$$


where $\kappa=\frac{C}{\rho g B}$.

In the common case where rock friction is described by associated plasticity, the angle of dilatancy $\psi$ is taken equal to the friction angle $\phi$, which means that the surface rugosity alone explains the friction along fault planes. In that case, the normalized overpressure $\Pi$ may be expressed as:

$$
\begin{array}{r}
\Pi=\frac{P-k \rho g H}{\rho g B}=(1-k) \lambda+\frac{1}{4} \tan \beta\left(\frac{\lambda}{\tan \beta}-1\right)^{2}\left(1-\frac{\tan (\beta-\phi)}{\tan (\beta+\phi)}\right) \\
+2 \kappa \cos \phi \frac{\lambda \cos ^{2} \beta \cos \phi+\sin ^{2} \beta \sin \phi}{\sin 2 \beta \sin (\beta+\phi) \cos (\beta-\phi)}
\end{array}
$$

Equations (16) and (20) show that overpressure may be decomposed in three contributions and allows an analysis of the pressurization and deformation process:

- a term $\Pi_{0}=(1-k) \lambda$ controlled by the pre-existing lithostatic pressure. This term is the amount of pressure necessary to reach, from the initial lithostatic pressure, the vertical equilibrium of the rock column of height $\mathrm{H}$ above the reservoir considering this one isolated (ruptured) from the host rock. It causes the reservoir volume increase and isotropic damage that precede the strain localization (formation of fault planes). It corresponds to the initial pressurization phase; during this phase there is no surface deformation, no free surface effect and the state of stress around the reservoir is compressive;

- a frictional term $\Pi_{f}=\frac{1}{4} \tan \beta\left(\frac{\lambda}{\tan \beta}-1\right)^{2}\left(1-\frac{\tan (\beta-\phi)}{\tan (\beta+\phi)}\right)$ which is a budget measured by the displacement of the block number 2; it corresponds to the overpressure necessary for the vertical, anisotropic, displacement and deformation along the fault plane structure 
that appears after strain localization. This term is controlled by the friction angle $\phi$ and it is zero when $\phi$ is null. It is also null for $\tan \beta=\tan \beta_{c}=\lambda$. It results from the friction along the fault plane structure after its creation, after the weakening of the volcanic edifice and the progressive opening of the eruptive/intrusive system; this term is dominant during large (vertical) deformation processes, especially immediately before, or during, eruptions or intrusions; it tends to be dominant during the final pressurization phase, and constitutes a minimum value for the pressure;

- a dissipative term $\Pi_{D}=2 \kappa \cos \phi \frac{\lambda \cos ^{2} \beta \cos \phi+\sin ^{2} \beta \sin \phi}{\sin 2 \beta \sin (\beta+\phi) \cos (\beta-\phi)}$ that corresponds to the (shortterm) irreversible part of the deformation. It is controlled by the $C \cos \phi$ term, that is, mostly by the cohesion $\mathrm{C}$ since $\phi$ keeps values in a relatively narrow interval for rocks, even at the scale of the rock mass. If $\mathrm{C}$ is null, no effort is spent in rock decohesion and this term vanishes. In this case minimal pressure corresponds to $\beta=\beta_{c}$, that is $W_{G}=0$ (no graben). Decohesion is associated to microseismicity and damage during the pre-eruptive/intrusive process; it leads progressively to strain localization when the free-surface effect appears. It precedes the phase of large displacement along the fault planes that are finally created. This term corresponds to the pressure necessary to create the fault plane structure, and to open the eruptive/intrusive system; it works during the intermediate phase between the initial and final pressurization phases described above. This is a transient contribution that decreases with cohesion and time during the preeruptive/intrusive process. In this work cohesion is used to describe the unconfined rock strength, but cohesion may also be easily related to tensile strength, which is more often used to quantify rock strength around magma reservoirs. Cohesion may eventually be 
low, at the scale of the rock mass, in fractured media. This term is generally considered alone to quantify the overpressure necessary for the rupture of magma reservoirs.

Therefore in this simple expression we retrieve the main phases of the deformation process, and their contribution to the magma reservoir pressure. It helps to understand how overpressure builds and may be used to describe the time evolution of this pressure, eventually from geophysical observables: seismicity and surface deformation. It highlights the multiple causes of overpressure, which may be the origin of contradictory interpretations [Gudmundsson, 2012; Grosfils, 2007].

Equation 20 and Figure 5 show that the overpressure $\Pi(\lambda)$ is a parabola with a first order term in $\lambda$ dependent on $\kappa$. For values of $\kappa$ larger than $0.5, \Pi$ is close to a linear function of $\lambda$ in the vicinity of $\tan \beta$ (close to 2 in Figure 5), that is, in the practical interval of interest for $\lambda$. The overpressure $\Pi$ is linearly dependent on $\kappa$ (equation 20). These results may be compared to those obtained by Haug et al. [2018], who found that overpressure was linearly dependent on the cohesion, and decreasing as a function of $\mathrm{B} / \mathrm{H}$ (inverse of $\lambda$ ) with a power law.

\subsection{Inferring the faulting structure from $\lambda=\frac{H}{B}$ ratio, cohesion and friction angle}

Expression (20) also contains information on the structure itself, and on its control parameters.

For a given reservoir position and size, and given rock mechanical properties, the fault plane structure is created in such a way that minimal effort, that is minimal pressure, is required. The only parameter of the structure that controls the pressure is the angle $\beta$. Minimization of the pressure with $\beta$ allows us to find the optimal angle for which 


$$
\begin{aligned}
\lambda & =\rho_{R} \tan \beta \\
& =\frac{1+\alpha}{1-\alpha} \tan \beta
\end{aligned}
$$

where $\rho_{R}=\frac{W_{D}+W_{G}}{W_{D}-W_{G}}$ and $\alpha=\frac{W_{G}}{W_{D}}$. As a consequence, $\alpha$ may be written:

$$
\alpha=\frac{\lambda-\tan \beta}{\lambda+\tan \beta}
$$

The ratio $\alpha$ is between 0 and 1 . From equation (21) we can write

$$
\frac{\tan \beta}{\lambda}=\frac{\tan \beta}{\tan \beta_{c}}=\chi=\frac{1-\alpha}{1+\alpha}
$$

where $\tan \beta_{c}=\frac{H}{B}=\lambda$.

From equations $(22,23)$ we infer that $\alpha$ is non-zero and the central graben exists if $\tan \beta<\lambda=\tan \beta_{c}$, that is, if the dissipation effect is non-zero. Therefore $\alpha$ and $\beta$ depend on $\lambda$ and $\kappa$ (Figures S4 and S5). From these results we can infer that the graben exists $(\alpha>0)$ for sufficiently high values of $\lambda$ and $\kappa$.

Figure 7 shows the variation of $\beta$ as a function of $\lambda$, for various values of $\kappa$ and $\phi$. It shows that the family of $\beta=f(\lambda)$ curves has a characteristic point $\left(\lambda_{0}, \beta_{0}\right)$ independent of $\kappa$; the family is comprised between two extreme curves corresponding to $\kappa=0$ (black line and crosses) and $\kappa \rightarrow \infty$ (red circles). The geometry, showing one central graben, studied by using limit analysis only exists for $\beta$ curves located below the black line, which corresponds to the limit case $\beta_{c}=\arctan (\lambda)$. This result shows that an increased cohesion induces a decrease in $\beta$ (see also Figure S5); it also shows that an increase in $\phi$ from 10 to 
$40^{\circ}$ induces a decrease of $\beta$ of at most $10^{\circ}$, which is reached for high cohesions and large $\lambda$ (Figure S6). The characteristic values $\left(\lambda_{0}, \beta_{0}\right)$ correspond to the minimal values of $\lambda$ (and $\beta$ ) for which the central graben exists; it is independent of the material cohesion.

Expressing the relation between $\beta_{0}$ and $\phi$ shows that $\beta_{0}=\frac{3 \pi}{8}-\frac{\phi}{2}$ for $15^{\circ}<\phi<75^{\circ}$ degrees (Figure S7).

The Figure S8 shows that $\tan \beta-\tan \beta_{0}$ is a linear function of $\lambda$ depending on $\kappa$ and $\phi$. A first-order approximation of this function leads to find the analytical expression (see Appendix 2 for details of the calculations):

$$
\tan \beta-\tan \beta_{0}=R(\kappa, \phi)\left(\lambda-\lambda_{0}\right)
$$

for $\lambda>\lambda_{0}$, where

$$
R(\kappa, \phi)=1-\frac{a_{0} \kappa}{c_{0} \kappa+d_{0}}\left(1+\frac{a_{1} \kappa+b_{1}}{c_{1} \kappa+d_{1}} \phi\right)
$$

$\lambda_{0}=\tan \beta_{0}=\tan \left(\frac{3 \pi}{8}-\frac{\phi}{2}\right)$, and $a_{0}, c_{0}, d_{0}, a_{1}, b_{1}, c_{1}, d_{1}$ are constants. Equation (24) may be considered as the first order Taylor's series expansion in $\phi$ for large values of $\phi$, of the solution found by optimization in $\beta$ of the equation (20). The first-order term $\frac{a_{1} \kappa+b_{1}}{c_{1} \kappa+d_{1}} \phi$ takes values in the $[-0.25,0.15]$ interval so that the dependency of $\beta$ on $\phi$ is relatively moderate in equation (24), and mostly due to the $\lambda_{0}$ term. However neglecting the firstorder term leads to significant changes for the highest values of $\kappa$. Increasing the order of the expansion enlarges the interval wherein the approximation is valid, at the expense of a larger number of parameters. The optimal order is found to be the first order, using a compromise between the parameter number and the goodness of fit. Results (Figure S9) show that $\beta$ is more strongly decreasing with the cohesive term $\kappa$. Using the values issued from our computations, we can infer that, when the central graben structure exists, for realistic rock mass characteristics $\left(\phi \approx 30^{\circ}\right), \beta$ grossly varies from about $50^{\circ}$ for $\lambda \approx 1$, to 
about $60^{\circ}$ for $\lambda \approx 2$ and $70^{\circ}$ for $\lambda \approx 6$ (Figure 7 ). These results are coherent with those obtained from analogue models, where values of 50 degrees $(\lambda=0.8$, Acocella et al. [2001]; Brothelande et al. [2016b]) and 60 degrees $(\lambda=2$, Acocella et al. [2001]) were found for $\beta$. The variation of $\beta$ with $\lambda$ is also coherent with the results obtained by Haug et al. [2018].

From $\tan \beta_{c}=\lambda=\rho_{R} \tan \beta$ and equation (24), we find that

$$
\begin{aligned}
\tan \beta & =\tan \beta_{0}+R(\kappa, \phi)\left(\lambda-\lambda_{0}\right) \\
& =(1-R(\kappa, \phi)) \tan \beta_{0}+R(\kappa, \phi) \rho_{R} \tan \beta
\end{aligned}
$$

where $\rho_{R}=\frac{W_{D}+W_{G}}{W_{D}-W_{G}}$ and $\lambda_{0}=\tan \beta_{0}=\tan \left(\frac{3 \pi}{8}-\frac{\phi}{2}\right)$; we infer:

$$
\tan \beta=\frac{1-R(\kappa, \phi)}{1-\rho_{R} R(\kappa, \phi)} \tan \beta_{0}
$$

Therefore $\rho_{R}=1$, that is $W_{G}=0$, corresponds to $\beta=\beta_{0}$ when $\kappa \neq 0$ (and $\beta=\beta_{c}=$ $\tan ^{-1}(\lambda)$ when $\left.\kappa=0\right)$.

Equation (27) has to verify the condition $\tan \beta_{c} \geq \tan \beta \geq \tan \beta_{0}$ for $\rho_{R} \geq 1$ (that is $\left.W_{G} \geq 0\right)$. The condition $\tan \beta \geq \tan \beta_{0}$ is true when $\rho_{R} \geq 1$ and $R(\kappa, \phi) \geq 0$, whatever can be $\lambda, \kappa, \phi$. The condition $\tan \beta \leq \tan \beta_{c}$ implies that $R(\kappa, \phi) \leq R(0, \phi)=\frac{\lambda-\lambda_{0}}{\rho_{R} \lambda-\lambda_{0}}$, so that equation (27) is valid when $0 \leq R(\kappa, \phi) \leq \frac{\lambda-\lambda_{0}}{\rho_{R} \lambda-\lambda_{0}} . R(\kappa, \phi)$ is always lower than $\frac{1}{\rho_{R}}$ when $\lambda$ tends to infinity. As $\rho_{R}$ is always larger than 1 , we can infer that $0 \leq R(\kappa, \phi) \leq 1$.

In volcanic environments, the angle $\beta$ is generally not directly found from field geological measurements, as inverse faults that reach the surface are more inferred from the deformation and topography rather then directly evidenced. Equation (27) therefore allows the estimation of $\beta$ from values of $\rho_{R}$, that is $W_{D}, W_{G}$ measured on the field, and estimates of $\kappa$ and $\phi$ determined at the scale of the rock mass. This estimation is used to compute $h_{c}$ and $r_{c}$, and the normalized overpressure terms $\Pi_{f}+\Pi_{D}$, since $\lambda=\rho_{R} \tan \beta$. We used our results to compute estimates of $\beta, h_{c}, r_{D Z}$ and $\Pi_{f}+\Pi_{D}$ in the case of the 
resurgent dome of Yenkahe (Vanuatu). From field and map measurements, we know that (see, e.g., Brothelande et al. $[2016 \mathrm{~b}]$ ) $W_{D}=2.3 \mathrm{~km}, W_{G}=0.6 \mathrm{~km}$ so that $\rho_{R}=1.7$; the corresponding interval of validity for the equation $(27)$ is $0 \leq R(\kappa, \phi) \leq 0.59$. For reasonable values of the friction angle $\phi$ comprised between $25^{\circ}$ and $45^{\circ}$, we find (Figure 8 ) that $60^{\circ} \leq \beta \leq 75^{\circ}, h_{c}$ comprised between $2.5 \mathrm{~km}$ and $4 \mathrm{~km}$, and $r_{D Z} \approx 1 \mathrm{~km}$. The radius of the magma reservoir is not directly known, as it depends on the damaged zone relative thickness $\Delta$, which is poorly known and likely to be comprised between 1 and 5 . Using a value of 2 to 3 for $\Delta$ allows us to find a magma reservoir radius of about 300 meters and a magma reservoir depth of about 2 to $3.5 \mathrm{~km}$, the depth being compatible with the numerical results of Brothelande et al. [2016b]. Variations of $h_{c}$ and $r_{D Z}$ with $W_{G}$ and $W_{D}$ are presented Figure S17; they show that $h_{c}$ is sensitive to variations in $W_{G}$ more than in $W_{D}$. Computation of the overpressure terms for Yenkahe within the intervals $25^{\circ} \leq \phi \leq 40^{\circ}$ and the corresponding $75^{\circ} \geq \beta \geq 60^{\circ}$ yields $0.06 \leq \Pi_{f} \leq 0.21$ and $3.26 \kappa \geq \Pi_{D} \geq 1.98 \kappa$; for $\phi=30^{\circ}$ and $\beta=65^{\circ}, \Pi_{f}=0.069$ and $\Pi_{D}=2.38 \kappa$. This result helps to quantify the fact that when the eruptive/intrusive system is closed or sealed (what happens after a long period of quiescence), the normalized overpressure required for the edifice rupture is $10 \kappa$ to $50 \kappa(0.4 \leq \kappa \leq 2)$ larger than the normalized overpressure needed when the edifice is already ruptured and deforms only by frictional sliding. From the former results, the overpressure involved in the crustal anisotropic deformation by the magma reservoir is close to $\rho g B \Pi_{D}$, that is approximately $2 C$. For cohesion values between 0.1 and $10 \mathrm{MPa}$, this part of the overpressure varies between 0.2 and $20 \mathrm{MPa}$. Computation of the isotropic $\rho g B \Pi_{0}=(1-k) \rho g H$ term shows that it scales with the depth $H$ of the magma reservoir, and reaches a value of $50 \mathrm{MPa}$ in the case of the Yenkahe dome. This order of magnitude 
is compatible with those computed by Grosfils [2007]. This isotropic overpressure term induces the isotropic damage around the magma reservoir. However in real cases where inflation/deflation cycles exist and correspond to time variation of the overpressure with irreversible strain in contraction, after the first cycle isotropic damage may pre-exist before the re-pressurization of the magma reservoir. In those cases it can be questioned if any depth-dependent overpressure is needed to localize the shear strain at the boundary of the damaged zone.

Values of reservoir radius and depth have been computed for some other resurgent domes (Table 1 and Figures S13-17) and may be compared to direct geophysical estimations. Campi Flegrei is the most well-investigated caldera. At this site, dome and graben halfwidths are taken from Sacchi et al. [2014], though it is difficult to know which graben structure is actually active nowadays; variations of the graben structure may indicate that depth and radius vary with time. At Campi Flegrei, most of these studies have estimated the source location to be beneath the Pozzuoli area at a depth around $3 \mathrm{~km}$ below sea level (e.g. Trasatti et al. [2008] and references therein; Bonafede et al. [1986]; Berrino et al. [1984]). The order of magnitude for the depth is corroborated by the seismicity recorded during the 1982-84 uplift that clustered between 1 and $4 \mathrm{~km}$ below sea level [Ferrucci et al., 1992]. Furthermore, seismic attenuation images provided by De Siena et al. [2010] indicate the presence of a small melt pocket beneath Pozzuoli located between 3 and 3.5 $\mathrm{km}$ depth. All these estimations match well with ours.

At Yellowstone, several tomography surveys have imaged a very large low-velocity zone, interpreted as a giant magma reservoir. Miller and Smith [1999] identified probable partial 
melting zones at about $8 \mathrm{~km}$ beneath the domes surface, a depth later confirmed by Husen et al. [2004] whereas Farrell et al. [2014] found a depth of about $6 \mathrm{~km}$.

At Valles caldera, resurgence is no longer active and probably finished more than 1 Myrs ago [Phillips et al., 2007]. The only direct geophysical constraint on the depth of the magma reservoir associated to resurgence is that it is greater than $3.25 \mathrm{~km}$, the maximum depth of the geothermal drilling campaign that did not penetrate any intrusive rock [Nielson and Hulen, 1984].

\section{Conclusion}

In this work we have investigated the deformation process around a pressurized magma reservoir embedded in a damaging elastic volcanic edifice. It has allowed us to evidence the action of the progressive damage process, and the structure created by the damage distribution. Damage is first isotropic around the cavity and constitutes a damaged zone. Then the free-surface effect appears and an anisotropic shear strain develops from the boundary of the damaged zone; it localizes on reverse faults that propagate upward to the surface. When the vertical surface deformation is sufficient, normal faulting appears. Finally, the complete structure shows an undeformed wedge above the top of the damaged zone, which strongly recalls the Prandtl's wedge. This structure is very similar to what is found by analogue modelling and from field geologic observations. From this model, we found a relation for reservoir radius and depth as a function of dome and graben widths. Magma reservoir pressure is deduced from limit analysis, which allows a better understanding of the magma pressure build-up. The dip of the reverse faults is inferred from the minimization of the pressure needed to rupture the crust around the magma reservoir. From that analysis, the magma reservoir overpressure, the dip of the faults, the 
Acknowledgments. This work was supported by grants from Université Savoie Mont Blanc (AO APS 2017) and European Research Council (ERC) under the European Union Horizon 2020 research and innovation program (Grant agreement no. 757848 CoQuake). This paper contains no data. 


\section{References}

Acocella, V., F. Cifelli, and R. Funiciello (2000), Analogue models of collapse calderas and resurgent domes, J. Volc. Geoth. Res., 104, 81-96.

Acocella, V., F. Cifelli, and R. Funiciello (2001), The control of overburden thickness on resurgent domes: insights from analogue models, J. Volc. Geoth. Res., 111, 137-153.

Amitrano, D. (2003), Brittle-ductile transition and associated seismicity: experimental and numerical studies and relationships with the b-value, J. Geophys. Res., 108, 20442059.

Amitrano, D., and A. Helmstetter (2006), Brittle creep, damage, and time to failure in rocks, J. Geophys. Res., 111, B11,201, doi:10.1029/2005JB004252.

Amitrano, D., J.-R. Grasso, and D. Hantz (1999), From diffuse to localized damage through elastic interaction, Geophys. Res. Lett., 26, 2109-2112.

Anderson, E. (1936), The dynamics of the formation of cone sheets, ring dikes and cauldron subsidence, Proc. R. Soc. Edinb., 56, 128-163.

Bailey, R., and G. Dalrymple (1976), Volcanism, structure, and geochronology of Long Valley caldera, Mono county, California, J. Geophys. Res., 81, 725-744.

Berrino, G., G. Corrado, G. Luongo, and B. Toro (1984), Ground deformation and gravity changes accompanying the 1982 Pozzuoli uplift, Bull. Volc., 47, 187-200.

Bigoni, D., and F. Laudiero (1989), The quasi-statica finite cavity expansion in a nonstandard elasto-plastic medium, Int. J. Mech. Sci., 31, 825-838.

Bonafede, M., M. Dragoni, and F. Quaroni (1986), Displacement and stress fields produced by a centre of dilation and by a pressure source in a viscoelastic halfspace: application to the study of ground deformation and seismic activity at Campi Flegrei, Italy, Geophys. 
J. R. Astr. Soc., 87, 455-485.

Brothelande, E., and O. Merle (2015), Estimation of magma depth for resurgent domes: An experimental approach, Earth Planet. Sci. Lett., 412, 143-151.

Brothelande, E., J. Lenat, M. Chaput, L. Gailler, A. Finizola, S. Dumont, and E. Garaebiti (2016a), Structure and evolution of an active resurgent dome evidenced by geophysical investigations: The Yenkahe dome-Yasur volcano system (Siwi caldera, Vanuatu), J. Volc. Geoth. Res., 322, 241-262.

Brothelande, E., A. Peltier, J.-L. Got, O. Merle, M. Lardy, and E. Garaebiti (2016b), Constraints on the source of resurgent doming inferred from analogue and numerical modeling Implications on the current feeding system of the Yenkahe domeYasur volcano complex (Vanuatu), J. Volc. Geoth. Res., 322, 225-240.

Carrier, A., J.-L. Got, A. Peltier, V. Ferrazzini, T. Staudacher, P. Kowalski, and P. Boissier (2015), A damage model for volcanic edifices: Implications for edifice strength, magma pressure, and eruptive processes, J. Geophys. Res., 120(1), 567-583.

Carter, J., J. Booker, and S. Yeung (1986), Cavity expansion in cohesive frictional soils, Geotechnique, 36, 349-358.

Chadwick, P. (1959), The quasi-static expansion of a spherical cavity in metals and ideal soils, Quart. J. Mech. Appl. Math., 12, 52-71.

Christensen, N., and W. Mooney (1995), Seismic velocity structure and composition of the continental crust: A global view, J. Geophys. Res., 100, 9,761-9,788.

Christiansen, R. (2001), The quaternary and Pliocene Yellowstone plateau volcanic field of Wyoming, Idaho, and Montana, USGS. Prof. Pap., 729-G. 
Das, S., and C. Scholz (1981), Theory of time-dependent rupture in the earth, J. Geophys. Res., 86, 6039-6051, doi:10.1029/2013JB002766.

Davis, R., and P. Selvadurai (2002), Plasticity and Geomechanics, Cambridge University Press.

Davison, I., M. Insley, M. Harper, P. Weston, D. Blundell, K. McClay, and A. Quillington (1993), Physical modelling of overburden deformation around salt diapirs, Tectonophysics, 228, 255-274.

de Borst, R. (2002), Fracture in quasi-brittle materials: a review of continuum damagebased approaches, Eng. Fract. Mech., 69, 95-112.

De Siena, L., E. Del Pezzo, and F. Bianco (2010), Seismic attenuation imaging of Campi Flegrei: Evidence of gas reservoirs, hydrothermal basins, and feeding systems, J. Geophys. Res., 115, 1-18.

D'Escatha, Y., and J. Mandel (1974), Stabilité d'une galerie peu profonde en terrain meuble, Extrait. Ind. Miner., pp. 45-53.

Drescher, A., and E. Detournay (1993), Limit load in translational failure mechanisms for associative and non-associative materials, Geotechnique, 43, 443-456.

Eberhardt, E., D. Stead, and J. Cogan (2004), Numerical analysis of initiation and progressive failure in natural rock slopesThe 1991 Randa rockslide, Int. J. Rock Mech. Min. Sci., 41, 69-87.

Farrell, J., R. B. Smith, S. Husen, and T. Diehl (2014), Tomography from 26 years of seismicity revealing that the spatial extent of the Yellowstone crustal magma reservoir extends well beyond the Yellowstone caldera, Geophys. Res. Lett., 41, 3068-3073. 
Ferrucci, F., A. Hirn, G. De Natale, J. Virieux, and L. Mirabile (1992), P-SV Conversions at a Shallow Boundary Beneath Campi Flegrei Caldera (Italy): Evidence for the Magma Chamber, J. Geophys. Res., 97, 351-359.

Fridrich, C., R. Smith, E. DeWitt, and E. McKee (1991), Structural, eruptive, and intrusive evolution of the Grizzly Peak caldera, Sawatch Range, Colorado, Geol. Soc. Am. Bull., 103, 1160-1177.

Galland, O., S. Planke, E. Neumann, and A. Malthe-Sorenssen (2009), Experimental modelling of shallow magma emplacement: Application to saucer-shaped intrusions, Earth Planet. Sci. Lett., 27r7, 373-383.

Gerbault, M., F. Cappa, and R. Hassani (2012), Elasto-plastic and hydromechanical models of failure around an infinitely long magma chamber, Geochem. Geophys. Geosyst., $13,1525-2027$.

Got, J.-L., A. Carrier, A. Marsan, V. Jouanne, K. Vogfjord, and T. Villemin (2017), An analysis of the nonlinear magma-edifice coupling at Grimsvtn volcano (Iceland), $J$. Geophys. Res. Solid Earth, 122, doi:10.1002/2016JB012905.

Grosfils, E. (2007), Magma reservoir failure on the terrestrial planets: assessing the importance of gravitational loading in simple elastic models, J. Volc. Geoth. Res., 166, $47-75$.

Gudmundsson, A. (2012), Magma chambers: formation, local stresses, excess pressures, and compartments, J. Volc. Geoth. Res., 166, 47-75.

Haug, O., O. Galland, P. Souloumiac, A. Souche, F. Guldstrand, T. Schmiedel, and B. Maillot (2018), Shear versus tensile failure mechanisms induced by sill intrusions Implications for emplacement of conical and saucer-shaped intrusions, J. Geophys. Res., 
45, 1099-1102, doi:10.1029/2018JB002766.

Heap, M., S. Vinciguerra, and P. Meredith (2009), The evolution of elastic moduli with increasing crack damage during cyclic stressing of a basalt from Mt. Etna volcano, Tectonophysics, 471, 153-160.

Heap, M., D. Faulkner, P. Meredith, and S. Vinciguerra (2010), Elastic moduli evolution and accompanying stress changes with increasing crack damage: implications for stress changes around fault zones and volcanoes during deformation, Geophys. J. Int., 183, $225-236$.

Hill, R. (1950), The mathematical theory of plasticity, Oxford University Press.

Hill, R., E. Lee, and S. Tupper (1947), The theory of combined plastic and elastic deformation with particular reference to a thick tube under internal pressure, Proc. R. Soc., A188, 278-303.

Hoek, E., and E. Brown (1997), Practical estimates of rock mass strength, Int. J. Rock Mech. Min. Sci. \& Geomech. Abstr., 34, 1165-1186.

Husen, S., R. Smith, and G. Waite (2004), Evidence for gas and magmatic sources beneath the Yellowstone volcanic field from seismic tomographic imaging, J. Volc. Geoth. Res., 131, 397-410.

Jeffery, G. (1920), Plane stress and plane strain in bipolar coordinates, Philos. Trans. $R$. Soc. London A, 221, 265-293.

Kachanov, L. M. (1958), Time of the rupture process under creep conditions, Isv. Akad. Nauk. SSR. Otd Tekh. Nauk, 8, 26-31.

Krajcinovic, D. (1996), Damage Mechanics, Elsevier. 
Lacroix, P., and D. Amitrano (2013), Long-term dynamics of rockslides and damage propagation inferred from mechanical modeling, J. Geophys. Res., 118, 1-16, doi: 10.1029/2013JB002766.

Lemaitre, J. (1994), Mechanics of solid materials, Cambridge University Press.

Lindsay, J., A. Schmitt, R. Trumbull, S. De Silva, W. Siebel, and R. Emmermann (2001), Magmatic evolution of the La Pacana caldera system, central Andes, Chile: compositional variation of two cogenetic, large-volume felsic ignimbrites, Jour. Petrology, 42, $459-486$.

Gardeweg, M., and C. Ramirez (1987), La Pacana caldera and the Atana Ignimbrite a major ash-flow and resurgent caldera complex in the Andes of northern Chile, Bull. Volcanol., 42, 459-486.

Lyakhovsky, V., Y. Ben-Zion, and A. Agnon (1997), Distributed damage, faulting, and friction, J. Geophys. Res., 102, 27,635-27,649.

Main, I. (2000), A damage mechanics model for power-law creep and earthquake aftershock and foreshock sequences, Geophys. J. Int., 142, 151-161.

Marti, J., G. Ablay, L. Redshaw, and R. Sparks (1994), Experimental studies of collapse calderas, J. Geol. Soc. London, 151, 919-929.

Merle, O., and B. Vendeville (1995), Experimental modelling of thin-skinned shortening around magmatic intrusions, Bull. Volcanol., 57, 33-43.

Merle, O., E. Brothelande, J.-F. Lénat, P. Bachélery, and E. Garaébiti (2013), A structural outline of the Yenkahe volcanic resurgent dome (Tanna Island, Vanuatu Arc, South Pacific), J. Volc. Geoth. Res., 268, 64-72. 
Miller, D., and R. Smith (1999), P and S velocity structure of the Yellowstone volcanic field from local earthquake and controlled source tomography, J. Geophys. Res., 104, $15,105-15,121$.

Nadai, A. (1931), Plasticity: Mechanics of the plastic state of matter.

Nadai, A. (1950), The theory of flow and fracture of solids.

Nielson, D., and J. Hulen (1984), Internal geology and evolution of the Redondo dome, Valles caldera, New Mexico, J. Geophys. Res., 89, 8695-8711.

Phillips, E. H., F. Goff, P. R. Kyle, W. C. McIntosh, N. W. Dunbar, and J. N. Gardner (2007), The $40 \mathrm{Ar} / 39$ Ar age constraints on the duration of resurgence at the Valles caldera, New Mexico, J. Geophys. Res., 112, B08,201.

Phillips, W. (1974), The dynamic emplacement of cone sheets, Tectonophysics, 24, 69-84.

Riva, F., F. Agliardi, D. Amitrano, and C. Crosta (2018), Damage-based time-dependent modeling of paraglacial to postglacial progressive failure of large rock slopes, J. Geophys. Res. Earth Surface, 123, doi:10.1002/2017JF004423.

Robson, G., and K. Barr (1964), The effect of stress on faulting and minor intrusions in the vicinity of a magma body, Bull. Volcanol., 27, 315-330.

Sacchi, M., F. Pepe, M. Corradino, D. Insigna, F. Molisso, and C. Lubritto (2014), The Neapolitan Yellow Tuff caldera offshore the Campi Flegrei: Stratal architecture and kinematic reconstruction during the last 15 ky, Mar. Geol., 354, 15-33.

Salencon, J. (1966), Expansion quasi-statique d'une cavite a symetrie spherique ou cylindrique dans un milieu elastoplastique, Annales des Ponts et Chaussees, 3, 175-197.

Sanford, A. (1959), Analytical and experimental study of simple geologic structures, Bull. Geol. Soc. Am., 237-238, 19-41. 
Scholz, C. (1972), Static fatigue of quartz, J. Geophys. Res., 77, 2104-2114.

Smith, R., and R. Bailey (1968), Resurgent cauldrons, Geol. Soc. Am. Mem., 116, 613662.

Smith, R., M. Jordan, B. Steinberger, C. Puskas, J. Farrell, G. Waite, S. Husen, W.-L. Chang, and R. O'Connell (2009), Geodynamics of the Yellowstone hotspot and mantle plume: Seismic and GPS imaging, kinematics, and mantle flow, J. Volc. Geoth. Res., $188,26-56$.

Souloumiac, P., B. Leroy, B. Maillot, and K. Krabbenhoeft (2009), Predicting stress distribution in fold-and-thrust belts and accretionary wedges by optimization, J. Geophys. Res., 114, 1-15, doi:10.1029/2008JB005986.

Steven, T., and P. Lipman (1976), Calderas of the San Juan Volcanic Field, Southwestern Colorado, U.S. Geol. Survey Prof. Paper, 958.

Sulem, J., M. Panet, and A. Guenot (1987), An analytical solution for time-dependent displacements in a circular tunnel, Int. J. Rock Mech. Min. Sci. Geomech. Abstr., 24, $155-164$.

Tibaldi, A. (2015), Structure of volcano plumbing systems: A review of multi-parametric effects, J. Volc. Geoth. Res., 298, 85-135.

Trasatti, E., F. Casu, C. Giunchi, S. Pepe, G. Solaro, S. Tagliaventi, and R. Lanari (2008), The 2004-2006 uplift episode at Campi Flegrei caldera (Italy): Constraints from SBASDInSAR ENVISAT data and Bayesian source inference, Geophys. Res. Lett., 35, 1-6.

Turcotte, D., and M. Glasscoe (2004), A damage model for the continuum rheology of the upper continental crust, Tectonophysics, 383, 71-80. 
Vardoulakis, I., J. Sulem, and A. Guenot (1988), Borehole instabilities as bifurcation phenomena, Int. J. Rock Mech. Min. Sci. Geomech. Abstr., 25, 159-170.

Vesic, A. (1972), Expansion of cavities in infinite soil mass, J. Soil. Mech. Fdns., 98, $265-290$.

Wiederhorn, S., and L. Bolz (1970), Stress corrosion and static fatigue of glass, J. Am. Ceram. Soc., 50, 543.

Yu, H. (2000), Cavity expansion methods in geomechanics, Kluwer Academic Publishers. 


\begin{tabular}{|l|l|r|r|r|r|r|r|}
\hline Caldera & Dome & $W_{D}(\mathrm{~km})$ & $W_{G}(\mathrm{~km})$ & $\rho_{R}$ & $\beta\left(^{\circ}\right)$ & $h_{c}(\mathrm{~km})$ & $r_{D Z}(\mathrm{~km})$ \\
\hline Siwi & Yenkahe & $1.5-2.2$ & $0.4-0.6$ & $1.5-2.3$ & $60-70$ & $2-4$ & $0.8-1$ \\
Valles & Redondo Dome & $4.5-4.9$ & $1.6-1.8$ & $2-2.3$ & $65-75$ & $9-12$ & 1.7 \\
Yellowstone & Mallard Lake & $4.5-5$ & $1.3-1.7$ & $1.7-2.2$ & $65-75$ & $9-12$ & $1.9-2$ \\
Creede & Snowshoe Mountain & $5.3-5.7$ & $0.9-1$ & $1.4-1.5$ & $60-65$ & $6.5-7.5$ & $2.6-2.7$ \\
La Pacana & Purifican-Bola & $5-5.5$ & $0.4-0.6$ & $1.15-1.3$ & $50-55$ & $5.5-6$ & $2.9-3.2$ \\
Campi Flegrei & Pozzuoli Bay & $2.2-2.6$ & $0.5-0.7$ & $1.5-1.9$ & $62-70$ & $2.5-3.5$ & 0.8 \\
\hline
\end{tabular}

Table 1. Results for the ratio $\rho_{R}=\frac{W_{D}+W_{G}}{W_{D}-W_{G}}$, the fault dip angle $\beta$, the reservoir depth $h_{c}$ and the damaged zone radius $r_{D Z}$ for various resurgent domes. Halfwidths of the dome $W_{D}$ and the graben $W_{G}$ are inferred from Smith and Bailey [1968] (Valles caldera), Smith et al. [2009] (Yellowstone), Steven and Lipman [1976] (Creede), Gardeweg and Ramirez [1987] (La Pacana), Sacchi et al. [2014] (Campi Flegrei, Figure 13). 

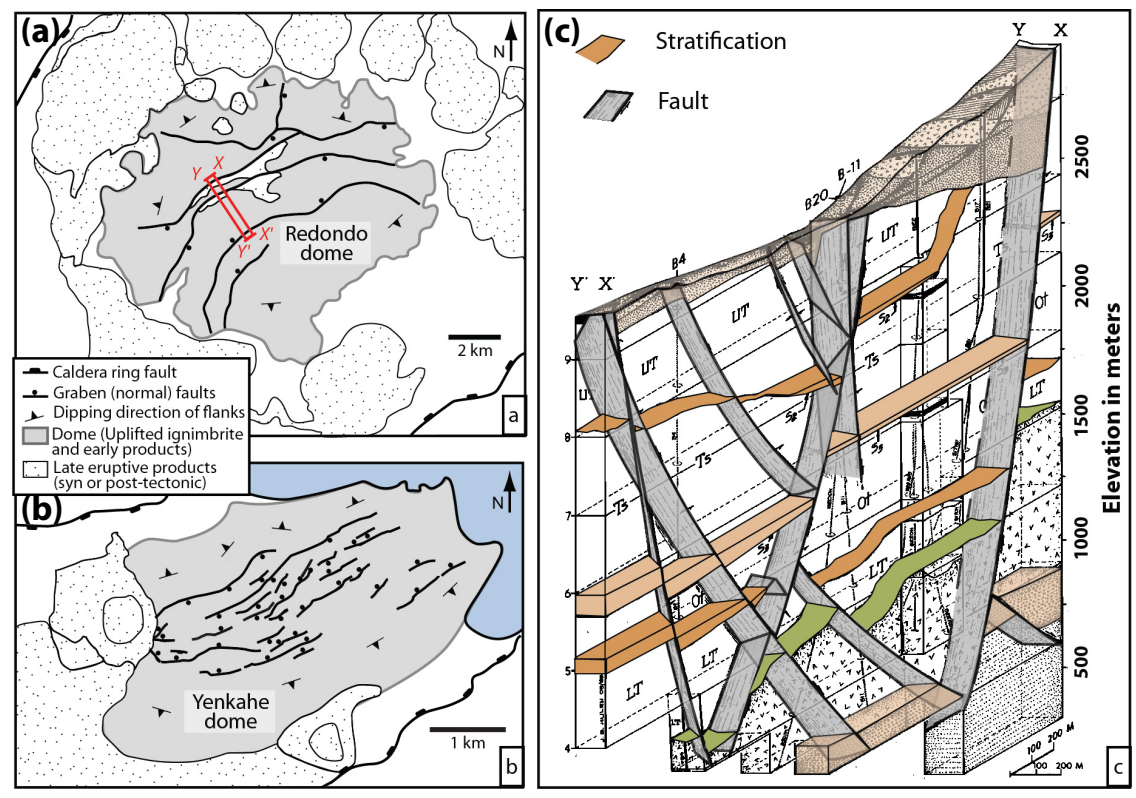

Figure 1. Simplified structural maps of (a) Redondo dome in Valles caldera (after Smith and Bailey [1968]) and (b) Yenkahe dome in Siwi caldera (after Brothelande et al. [2016a]). (c) Reconstructed 3D structure of the graben along $X X^{\prime} Y^{\prime} Y$ inferred from geothermal drilling (modified after Nielson and Hulen [1984]). 


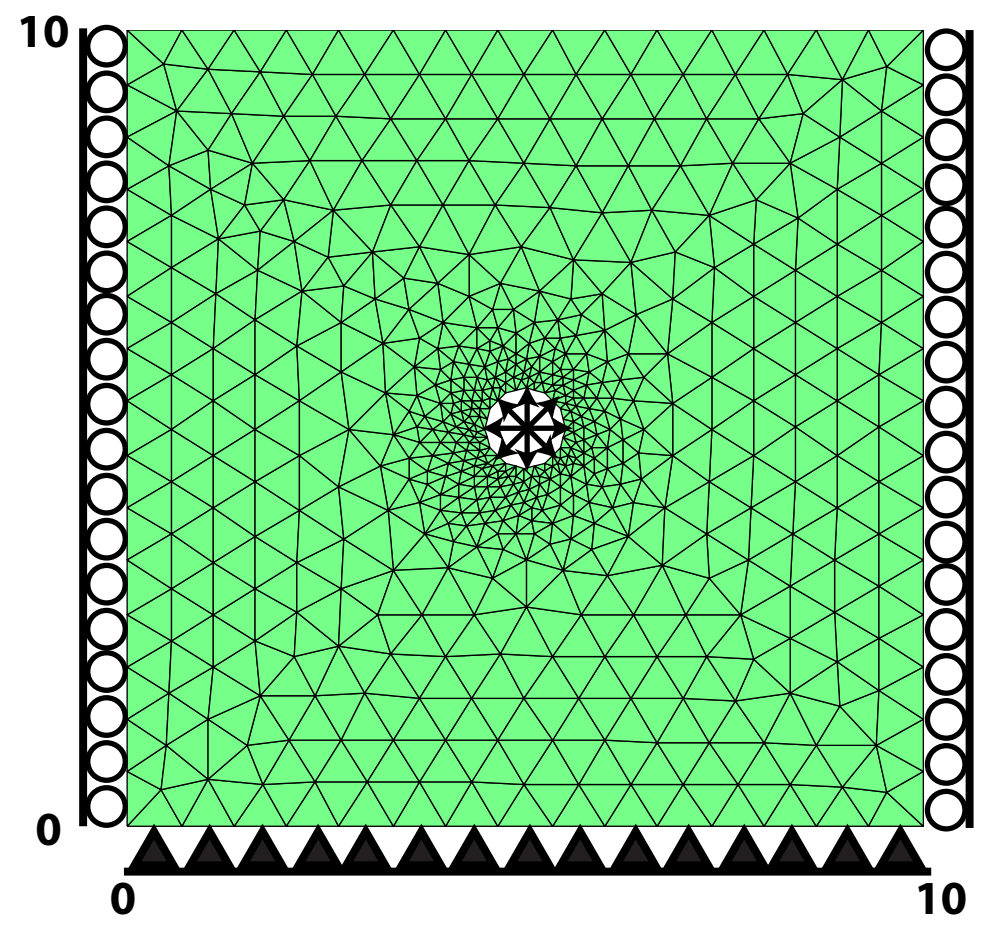

Figure 2. Model geometry and boundary conditions. 
(a)
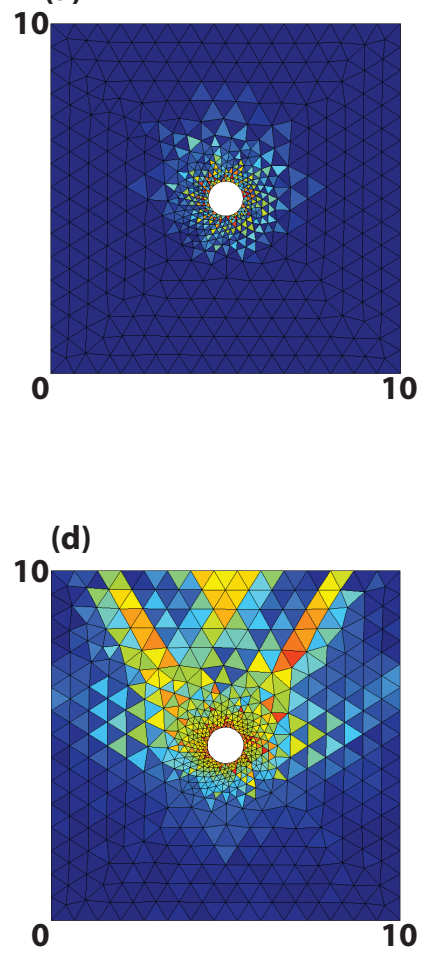

(b)

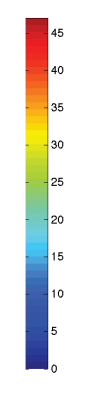

10

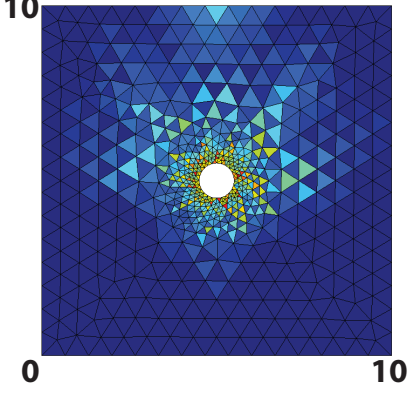

10
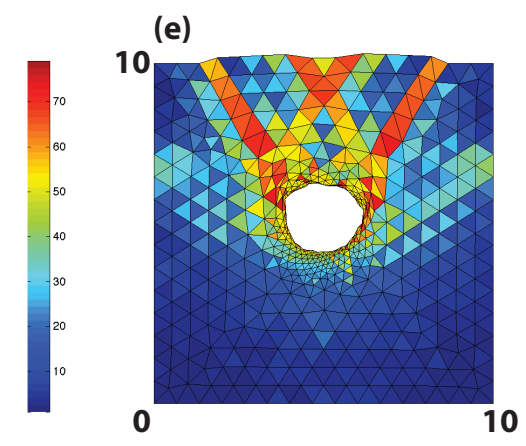
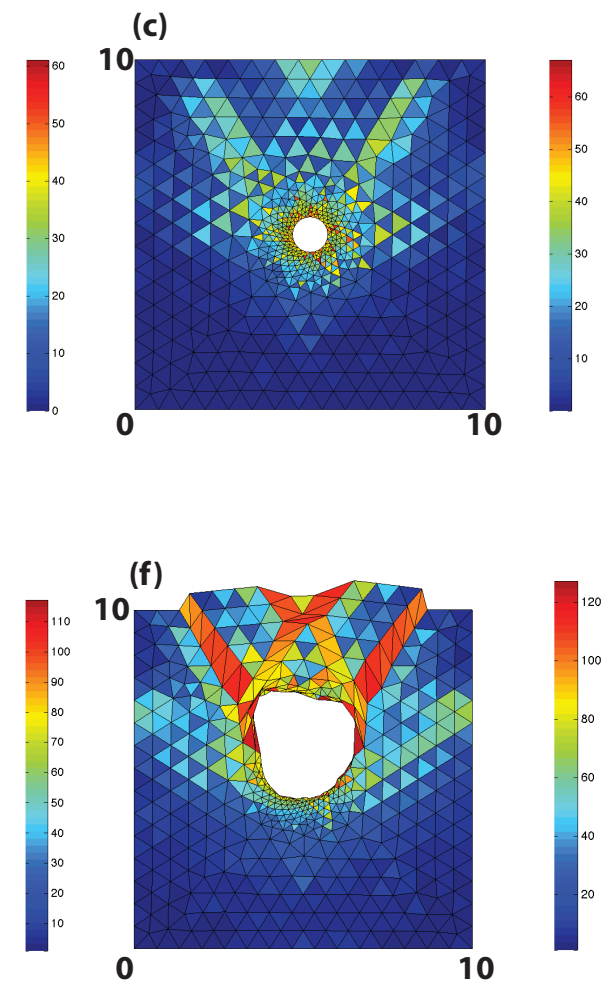

Figure 3. Number of damage events as a function of their position in the model at six stages of the strain localization process, showing the free-surface effect. 
(a)
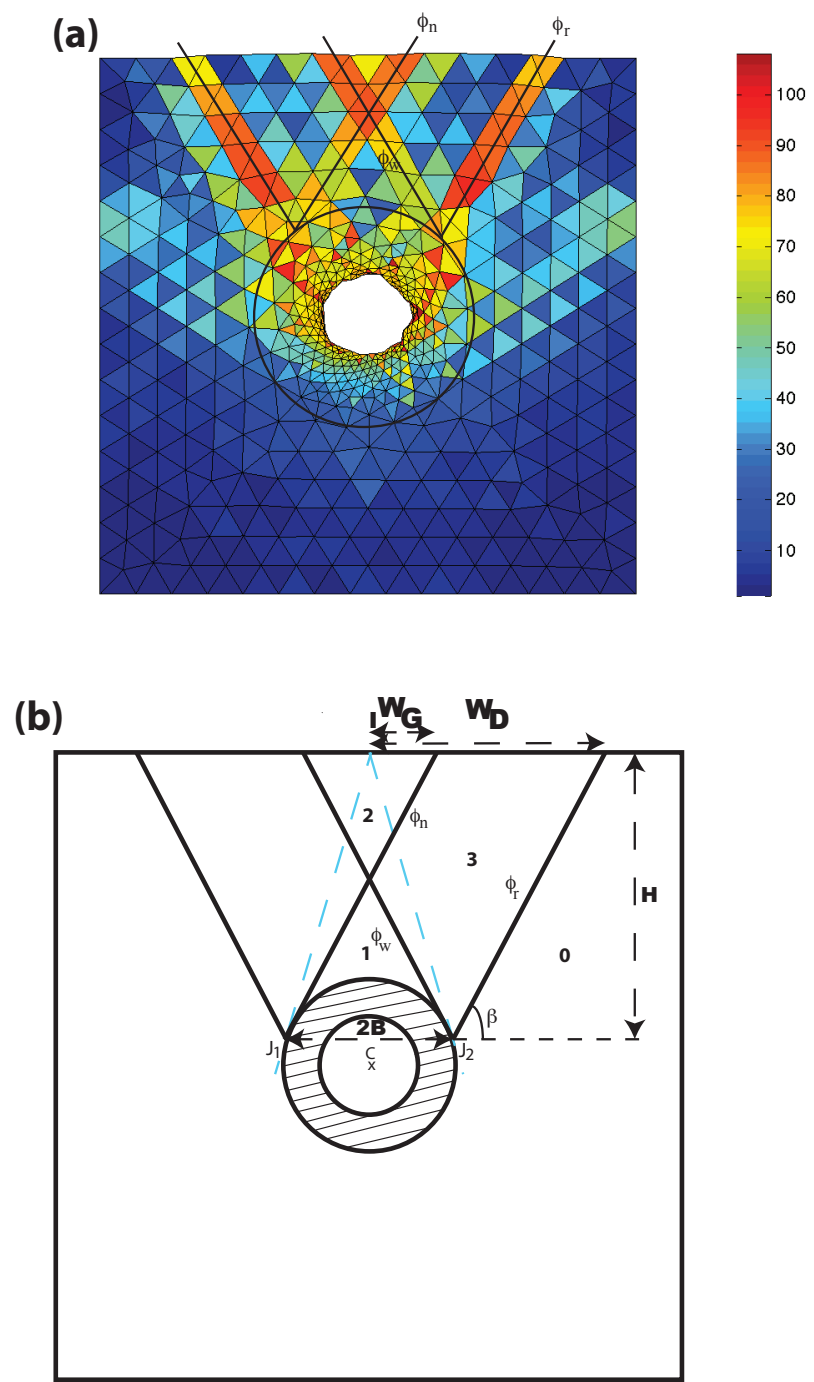

Figure 4. (a) Damage distribution around the pressurized magma reservoir, showing the main structural elements: damaged zone (inside the black circle), reverse faults $\left(\phi_{r}\right)$, normal faults $\left(\phi_{n}\right)$, undeformed Prandtl's wedge limited by the $\phi_{w}$ faults. (b) Model of the structure used for the limit analysis study, showing the damaged zone (hatched area), normal and reverse faults $\left(\phi_{n}, \phi_{r}, \phi_{w}\right)$, the blocks they delimit (numbers), and the graben and dome extent with their halfwidths $W_{G}$ and $W_{D}$. Block number 0 corresponds to the fixed, stable, structure; block number 1 corresponds to the Prandtl's wedge, above the magma reservoir; block number 2 corresponds to the graben; block number 3 corresponds to the part of the dome delimited by the normal $\left(\phi_{n}\right)$ and reverse $\left(\phi_{r}\right)$ faults. Blue dashed lines are tangent to the circle and used to find the Jeffery's points $J_{\mathrm{R}}, J_{2} ; J_{1} J_{2}=2 B$. The point $\mathrm{I}$ is at the vertical of the center $\mathrm{C}$ of the reservoir. $\mathrm{D}$ A $\mathrm{A} \mathrm{F}$ 


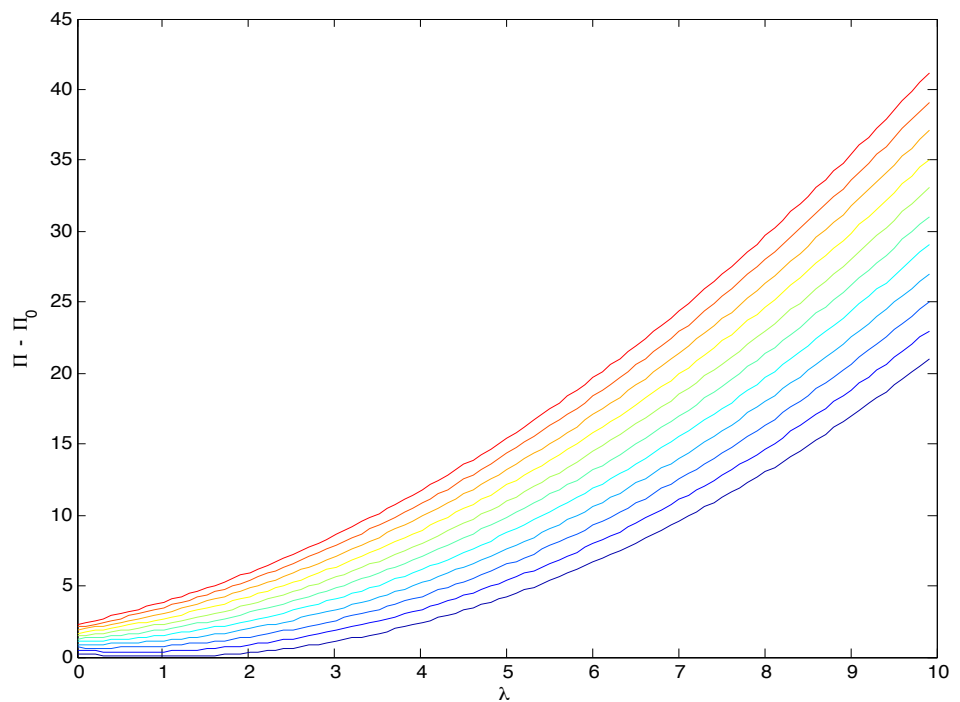

Figure 5. Overpressure $\Pi-\Pi_{0}=\Pi_{f}+\Pi_{D}$ (see text for details) in the cavity as a function of $\lambda$, for values of $\kappa$ varying between 0 (dark blue) and 2 (dark red) by step of 0.2 , with $\phi=30$ degrees, and $\beta=61$ degrees. 


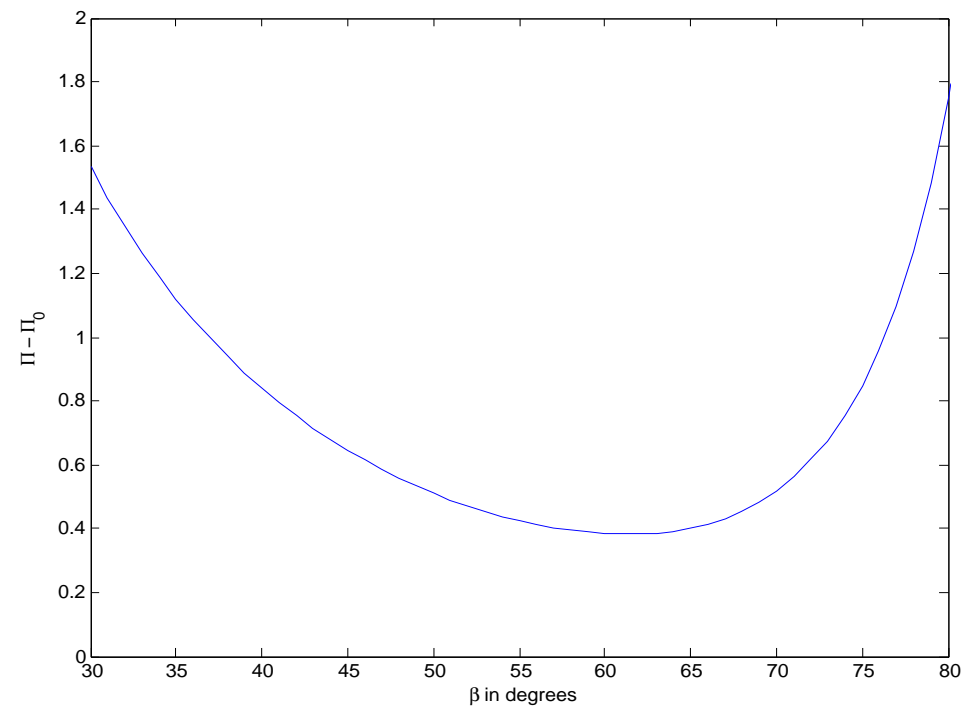

Figure 6. Overpressure $\Pi-\Pi_{0}=\Pi_{f}+\Pi_{D}$ (see text for details) in the cavity as a function of the angle $\beta$, for $\kappa=0.2, \lambda=2, \phi=30$ degrees. 

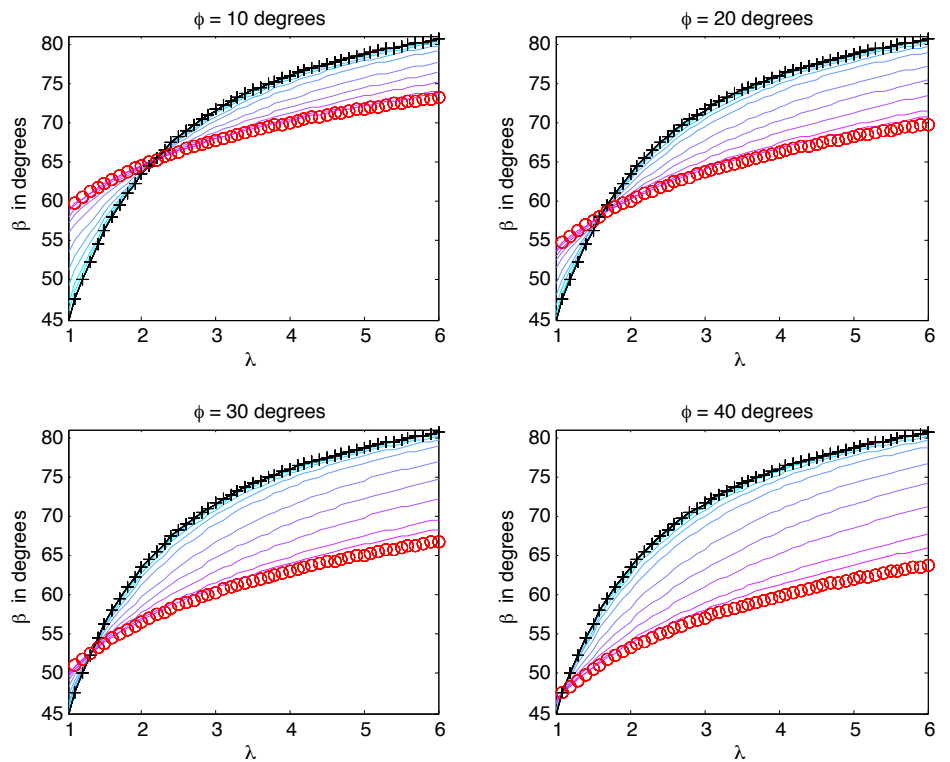

Figure 7. Angle $\beta$ as a function of $\lambda$, for various values of $\kappa(0.01,0.02,0.05,0.1,0.2,0.5$, $1,2,5,10$, from light blue to magenta), and various values of $\phi$; black crosses correspond to a purely frictional material $(\kappa=0)$, and red circles to a cohesive-frictional material with very high cohesion $(\kappa=100)$; black line (superposed to black crosses) corresponds to $\beta=\beta_{c}=\arctan (\lambda)$. (a): $\phi=10^{\circ} ;(\mathrm{b}): \phi=20^{\circ} ;(\mathrm{c}): \phi=30^{\circ} ;(\mathrm{d}): \phi=40^{\circ}$. The characteristic point, intersection of all curves, corresponds to $\beta_{0}=\frac{3 \pi}{8}-\frac{\phi}{2}$ (see text and Figure S7). 

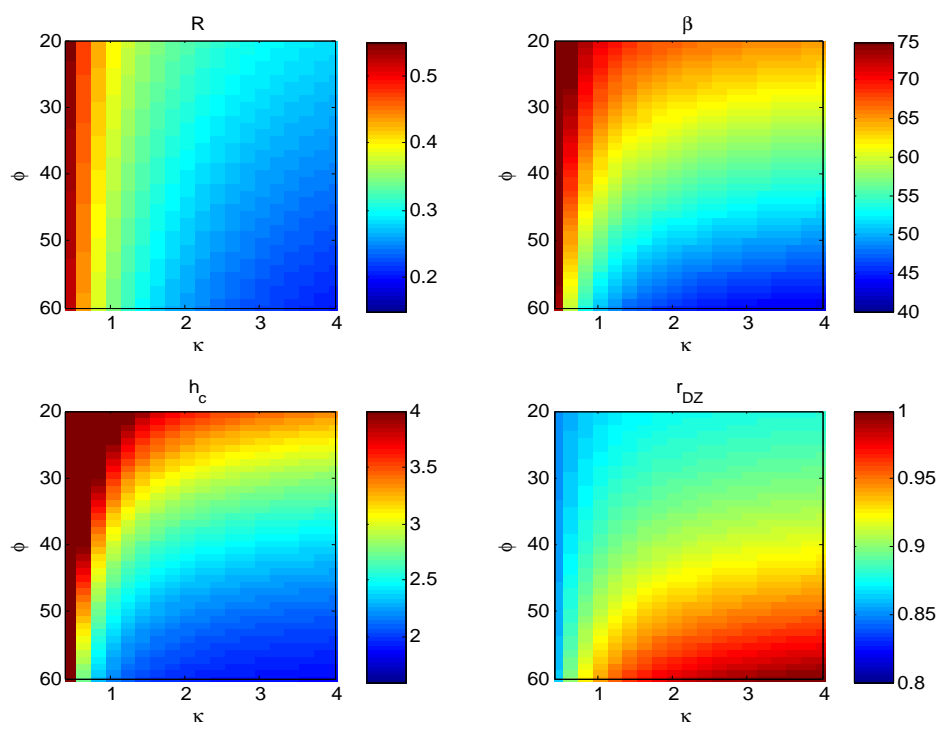

Figure 8. Results obtained for the Yenkahe caldera. (a): Function $R(\kappa, \phi)$ (Equation $(25)$ ); (b): Angle $\beta$ (Equation (27)) in degrees; (c) Depth $h_{c}$ (Equation (12)) in $\mathrm{km}$; (d) Radius of the damaged zone $r_{D Z}$ (Equations (13-14)) in $\mathrm{km}$, as a function of $\kappa$ and $\phi$ (in degrees). Values of $\beta, h_{c}$ and $r_{D Z}$ in the text corresponds to $25^{\circ} \leq \phi \leq 45^{\circ}$ and $\kappa \approx 1$. 
Figure1. 

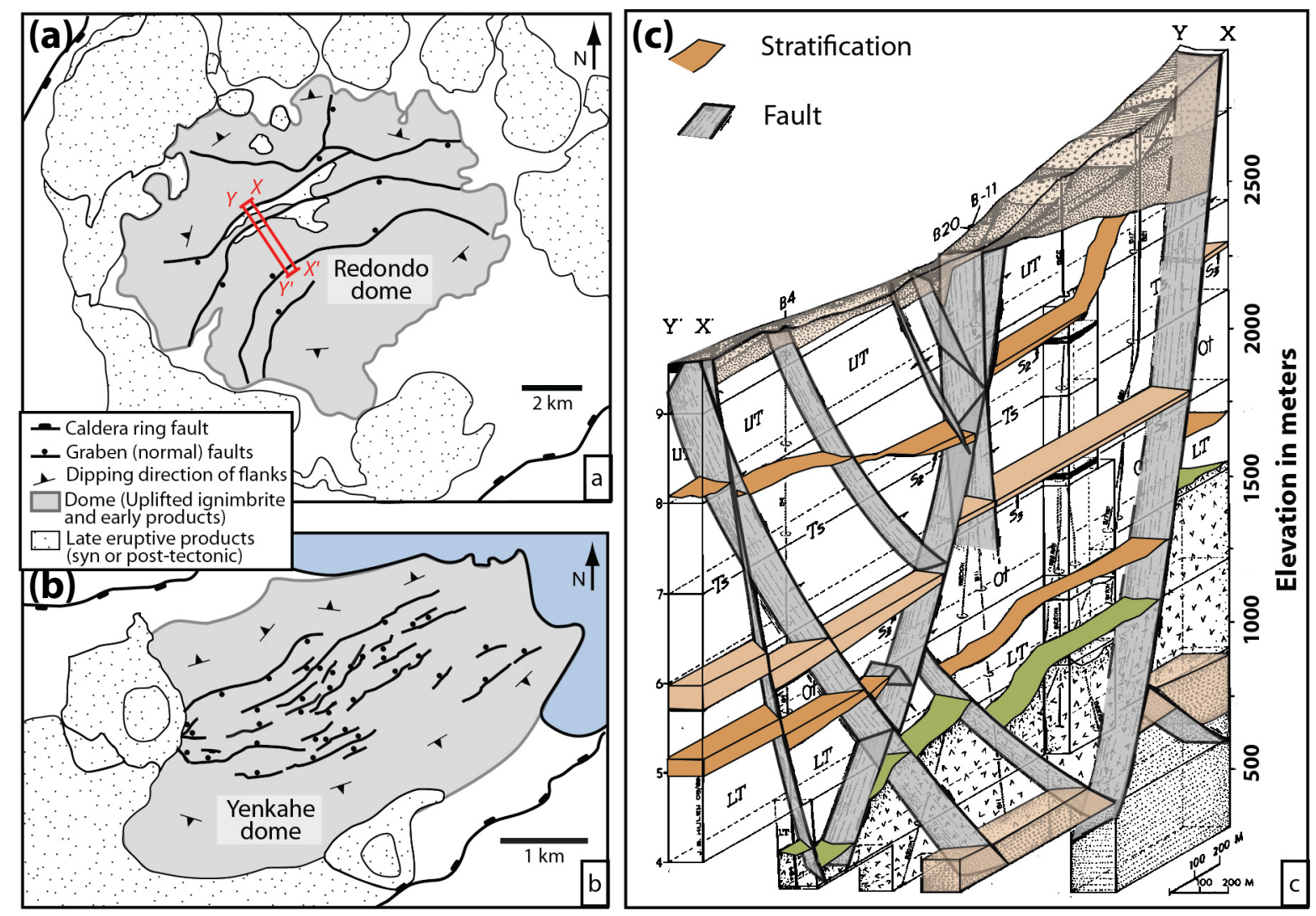
Figure2. 


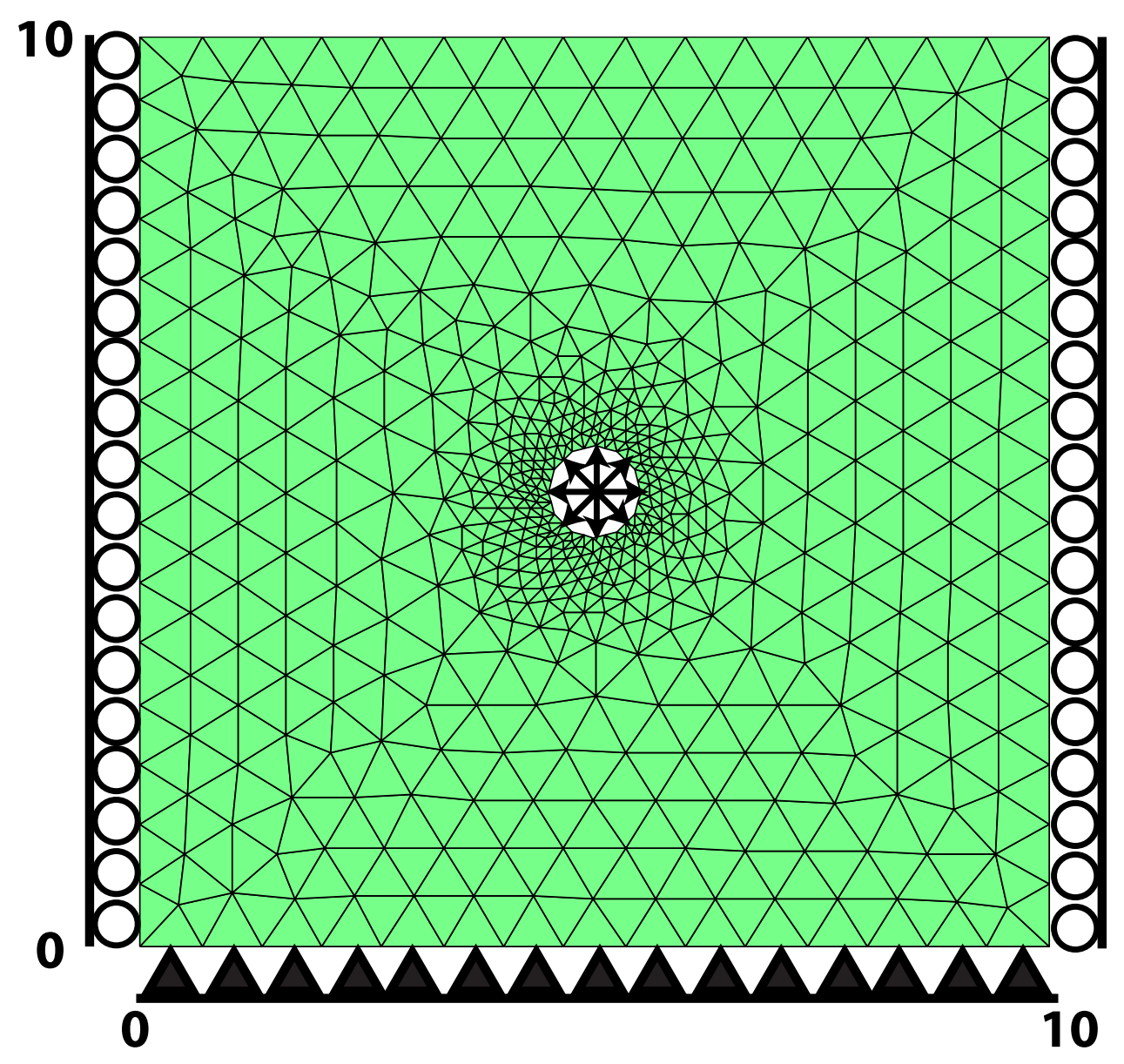


Figure3. 
(a)

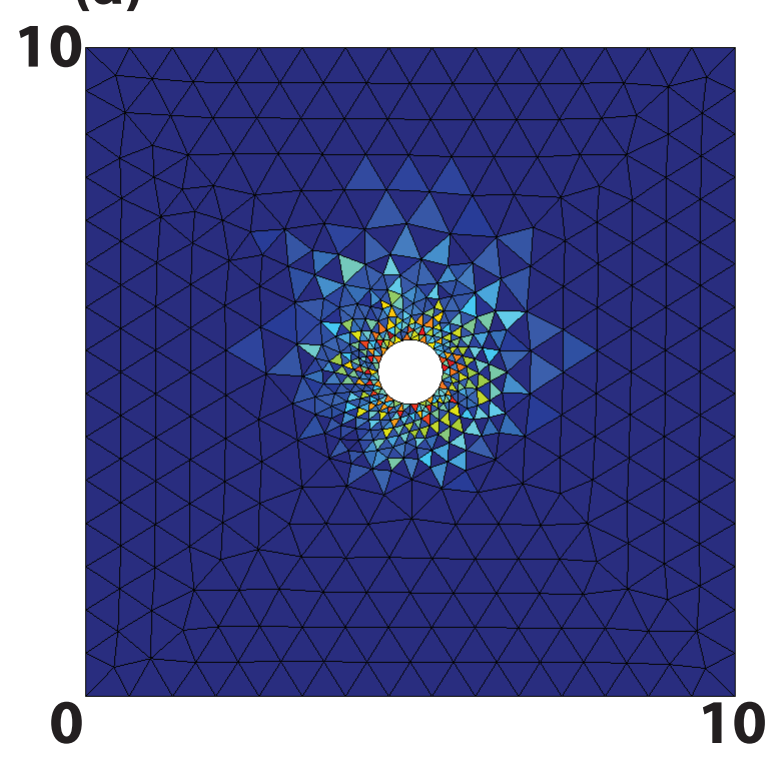

(d)

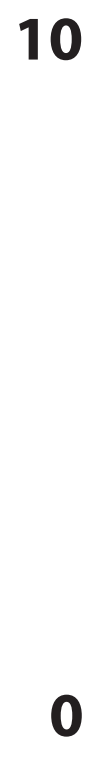

(b)

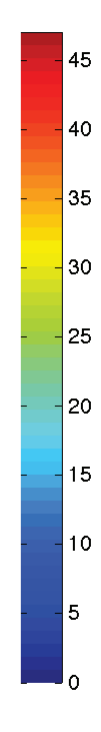

(c)
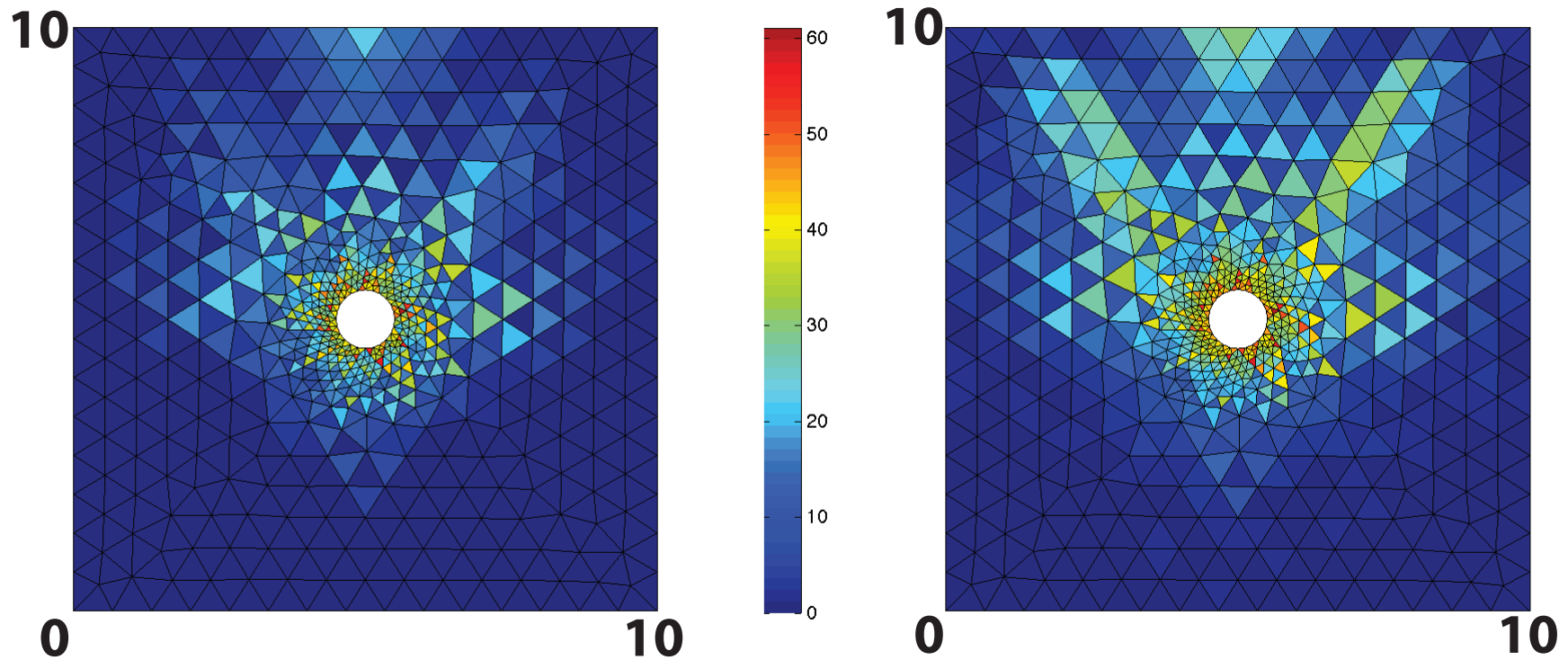

$-\int^{-60}-40$
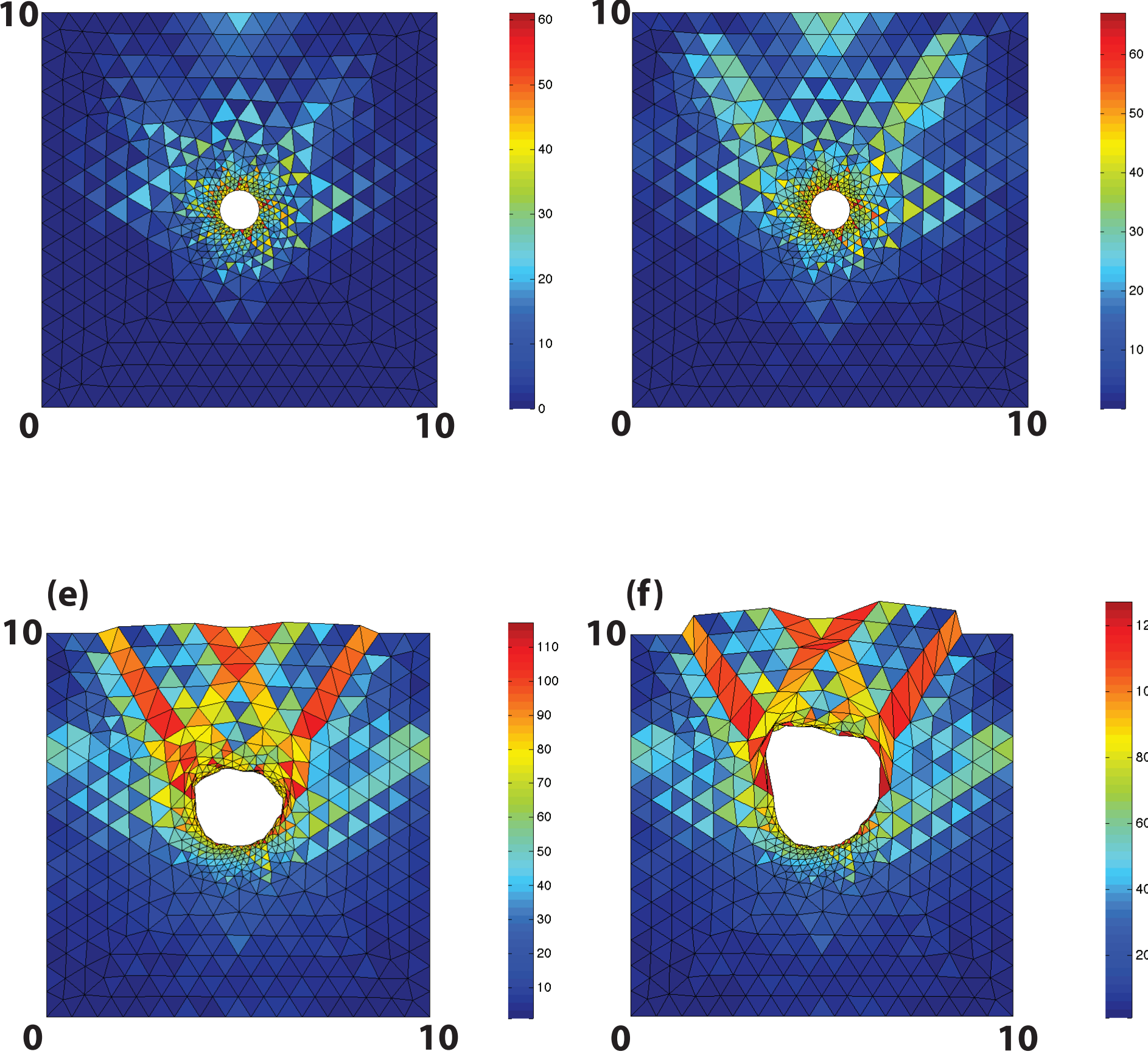

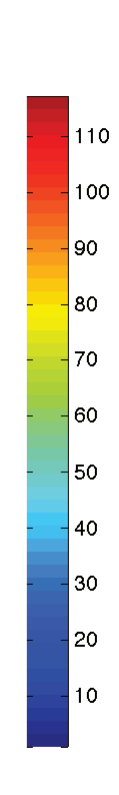

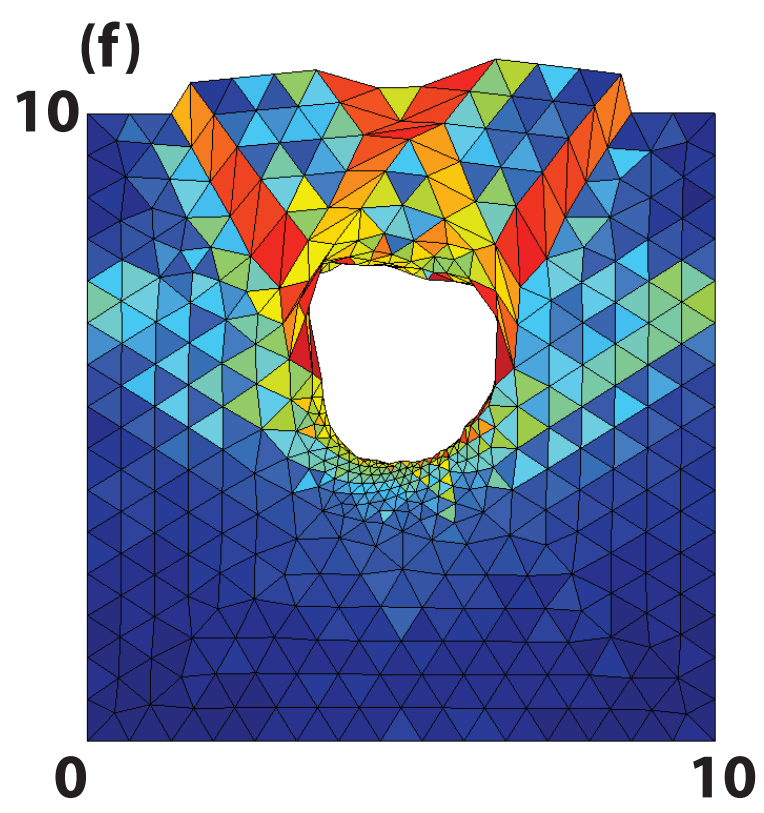

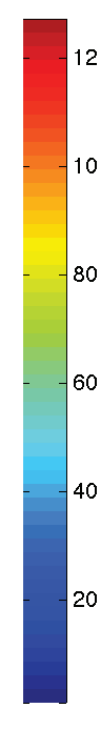


Figure4. 
(a)

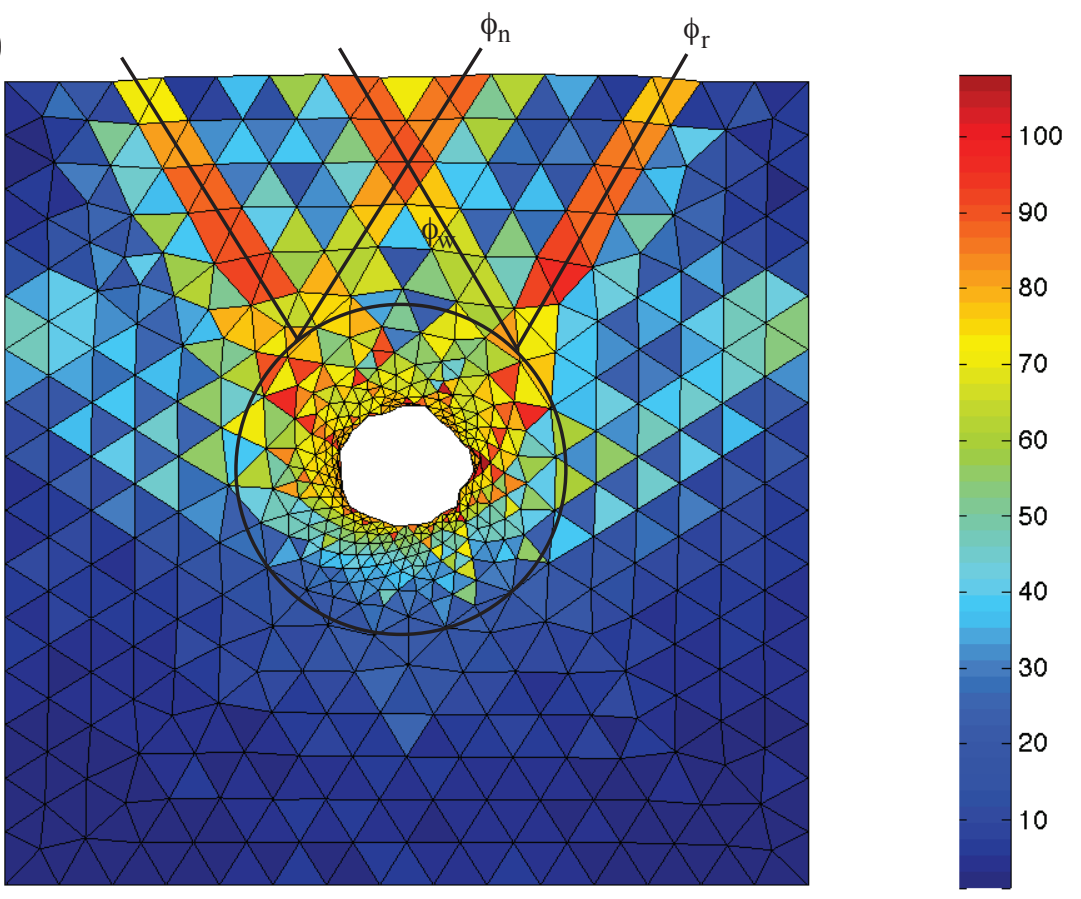

(b)

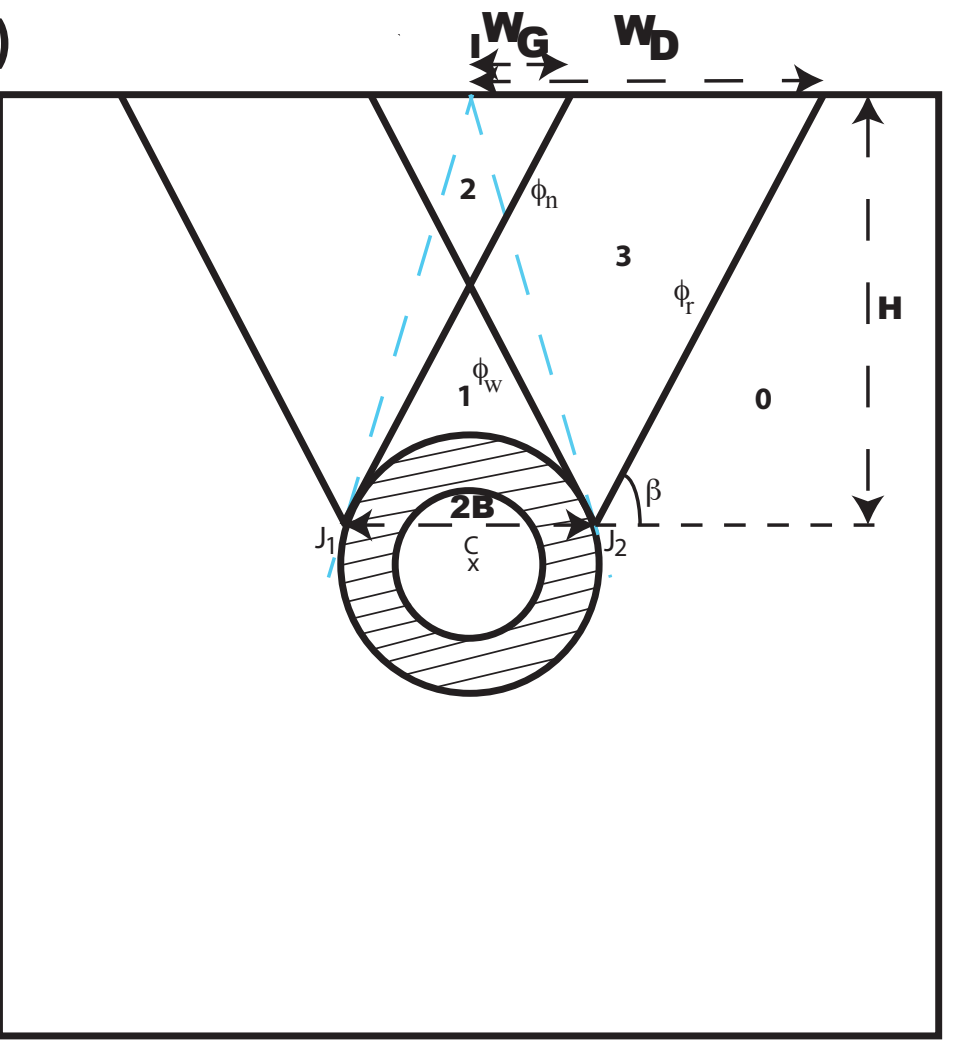


Figure5. 


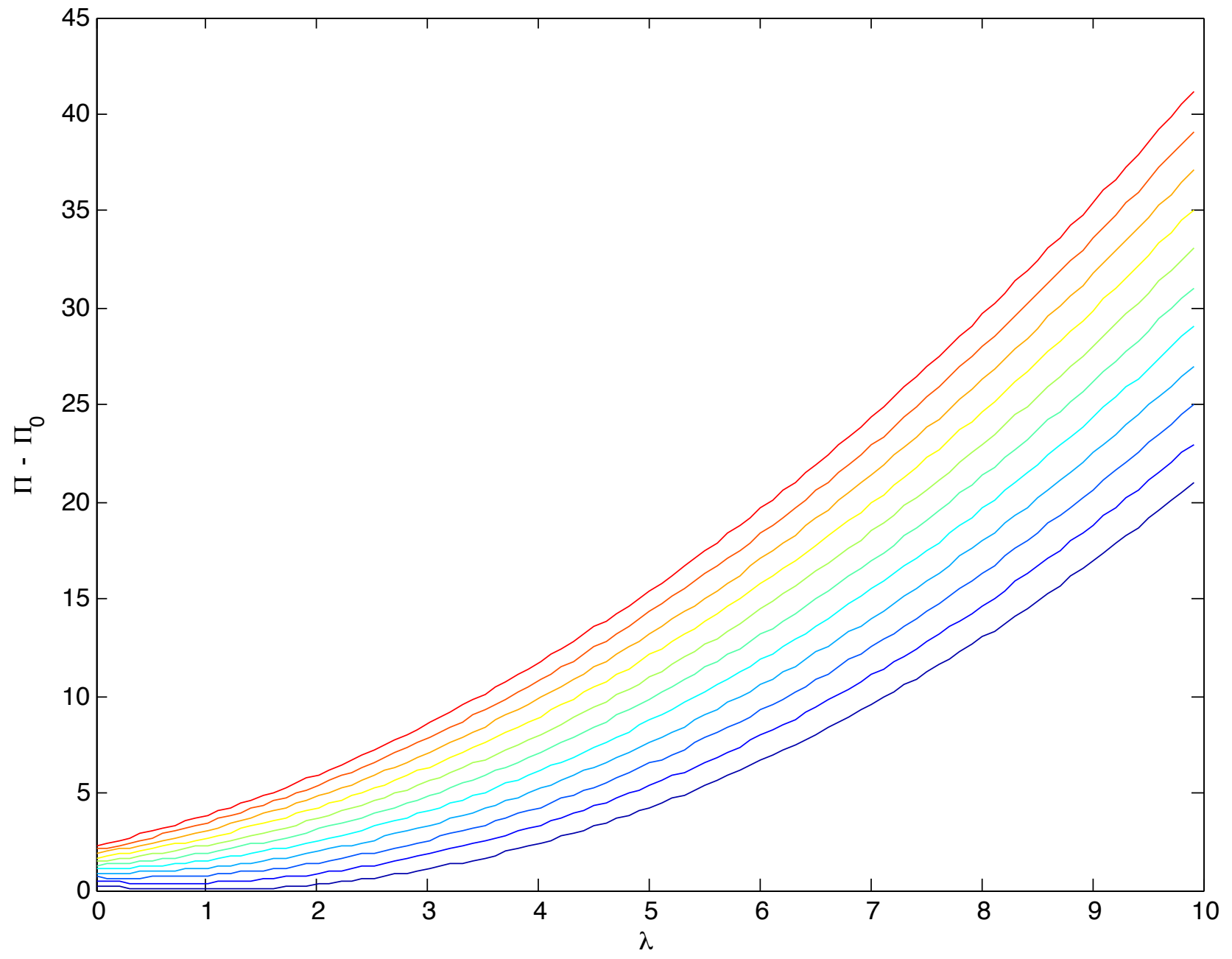


Figure6. 


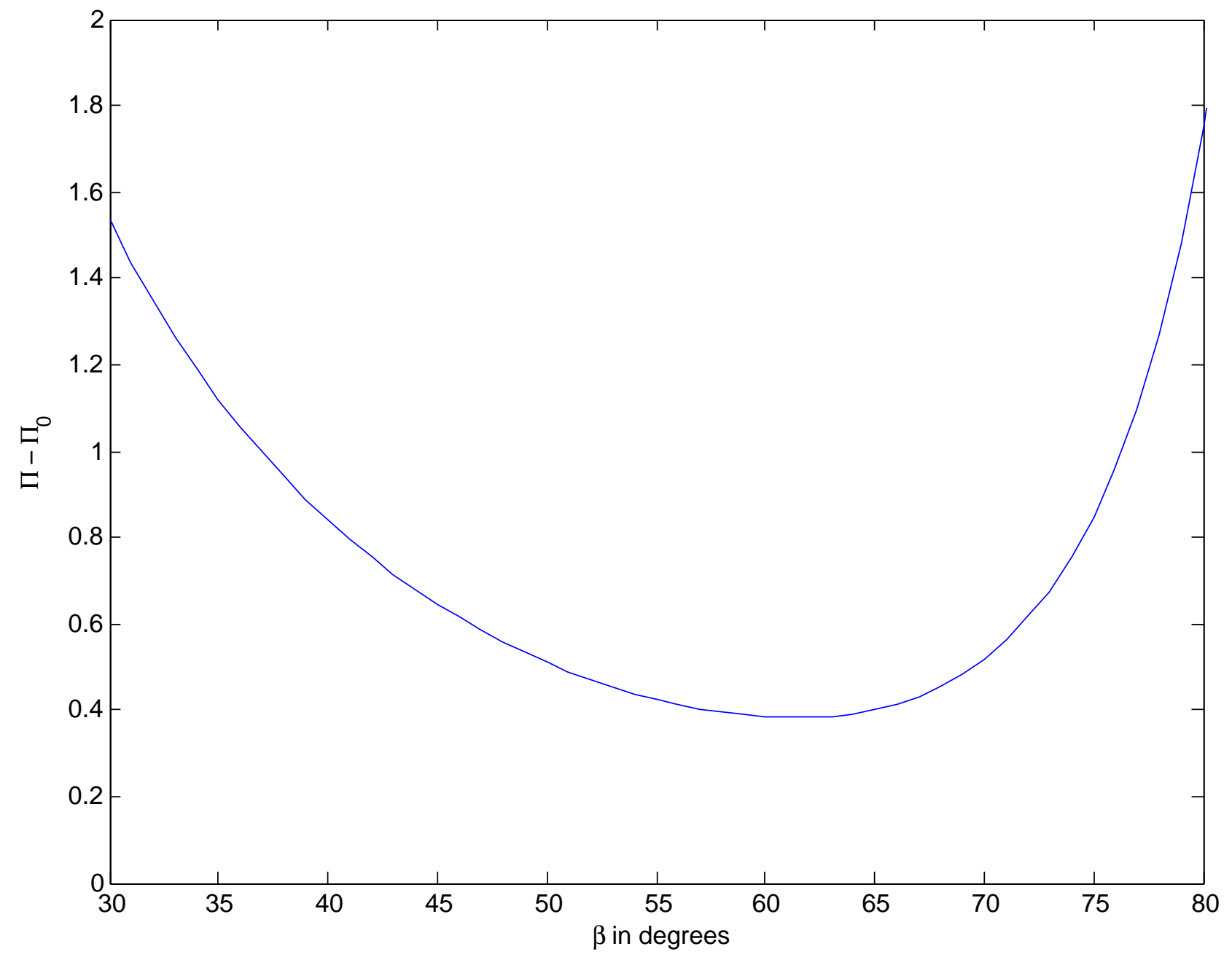


Figure7. 

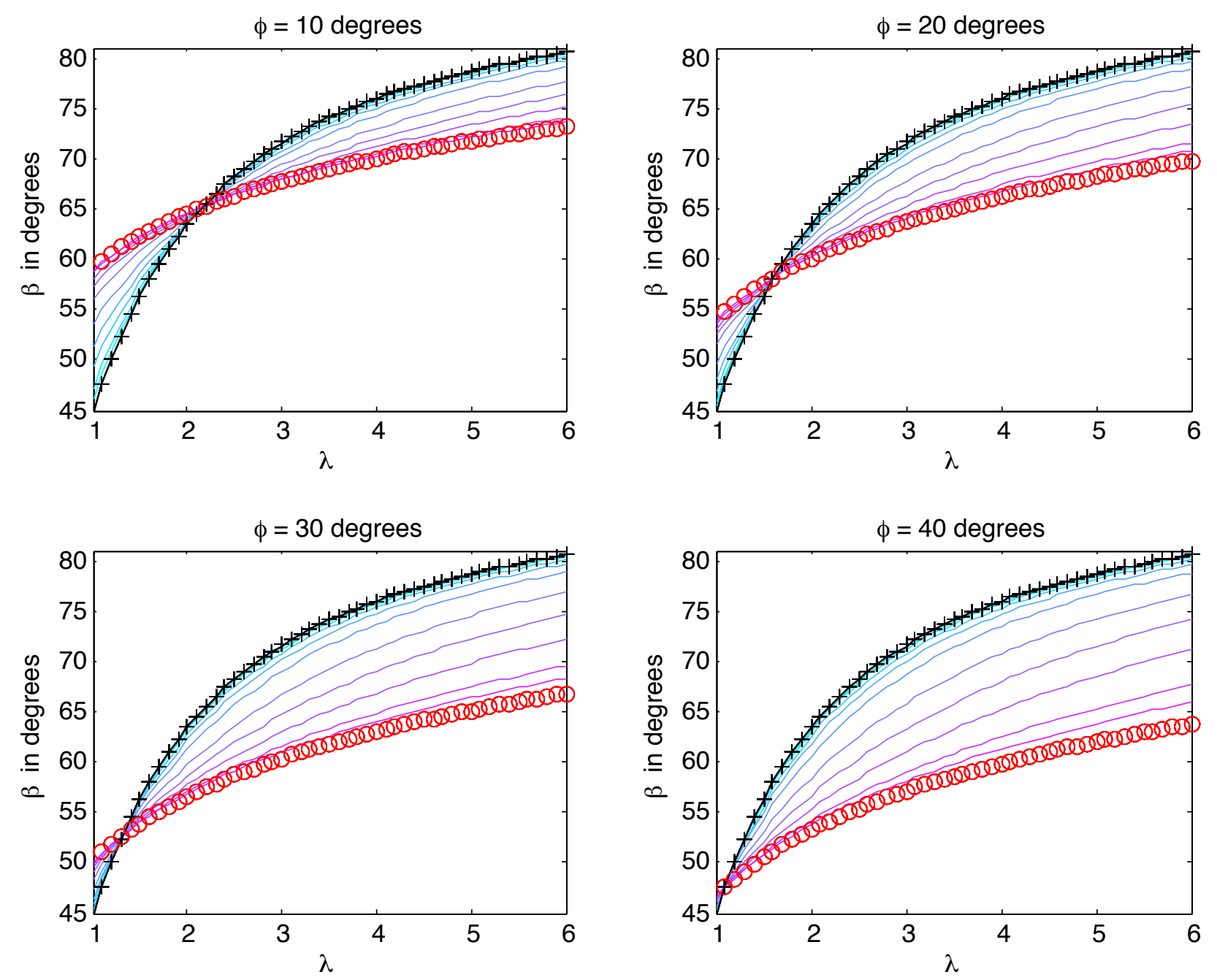
Figure8. 

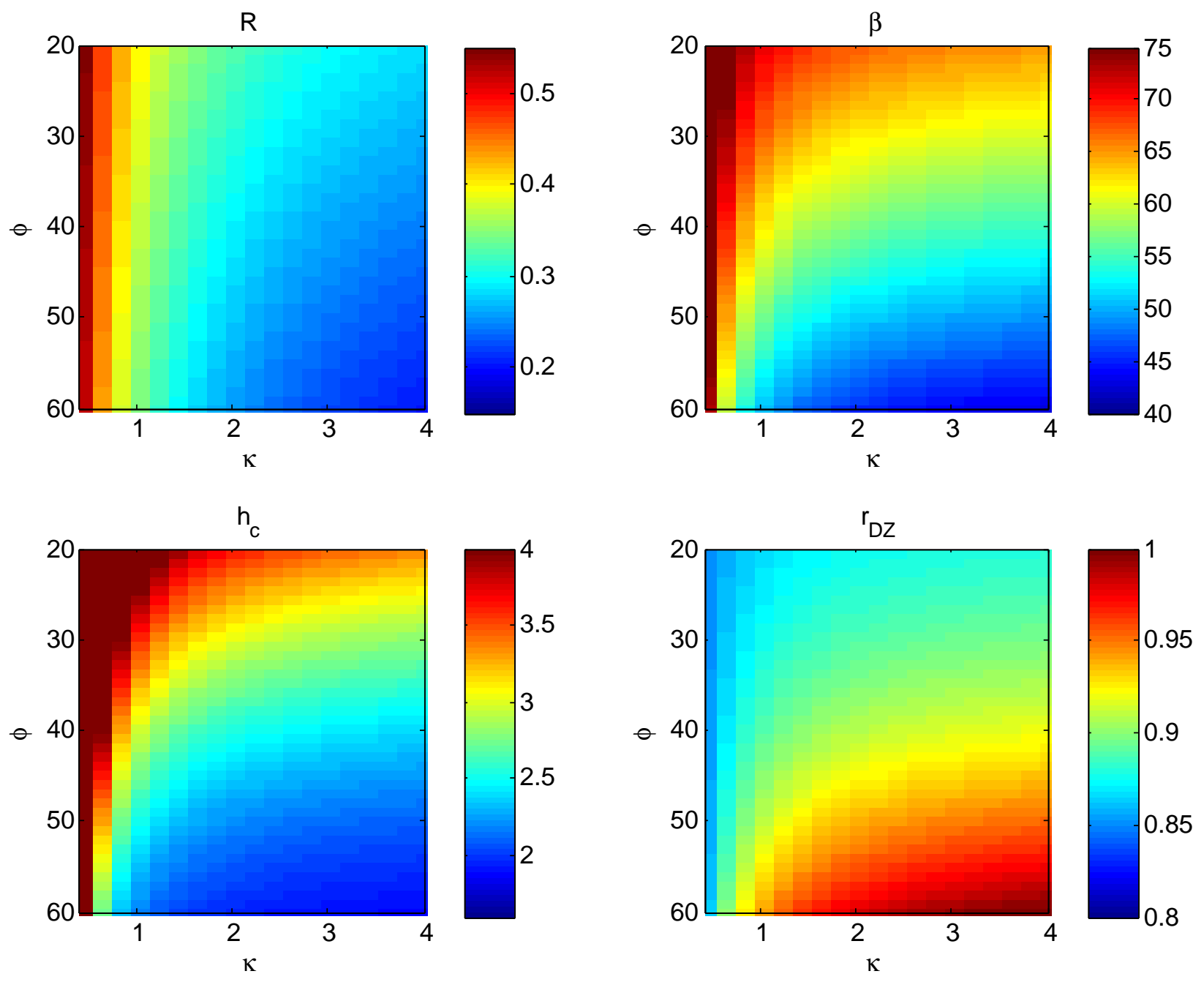